\title{
TRANSITION STATE SPECTROSCOPY OF BIMOLECULAR REACTIONS USING NEGATIVE ION PHOTODETACHMENT
}

\author{
RICARDO B. METZ, ${ }^{*}$ STEPHEN E. BRADFORTH, \\ and DANIEL M. NEUMARK $\dagger$ \\ Department of Chemistry, University of California, Berkeley, California
}

\section{CONTENTS}

I. Introduction

II. Experimental Methods

III. Theoretical Methods

A. Time-Independent Analysis

B. Time-Dependent Analysis

IV. Symmetric Hydrogen Exchange Reactions

A. Photoelectron Spectrum of $\mathrm{BrHBr}^{-}$

1. Summary of Results

2. Analysis: Preliminary Considerations

3. One-Dimensional Simulation of $\mathrm{BrHBr}^{-}$Spectrum

4. Two-Dimensional Simulation (Time-Independent)

5. Time-Dependent Analysis of the $\mathrm{BrHBr}^{-}$Photoelectron Spectrum

6. Discussion of Results

B. Photoelectron and Threshold Photodetachment Spectra of $\mathrm{IHI}^{-}$

1. Photoelectron Spectroscopy of $\mathrm{IHI}^{-}$and $\mathrm{IDI}^{-}$

2. Threshold Photodetachment Spectroscopy of $\mathrm{IHI}^{-}$

V. Asymmetric Hydrogen Exchange Reactions

A. General Considerations

B. Photoelectron Spectroscopy of $\mathrm{BrHI}^{-}$

1. Results and Qualitative Analysis

2. Time-Dependent Simulations of the Spectra

3. Excited Electronic States in the $\mathrm{BrHI}^{-}$Photoelectron Spectrum

C. Photoelectron Spectroscopy of $\mathrm{FHI}^{-}$and $\mathrm{FHBr}^{-}$

D. Photoelectron Spectroscopy of $\mathrm{CH}_{3} \mathrm{OHF}^{-}$and $\mathrm{CH}_{3} \mathrm{ODF}^{-}$

*University Fellow, University of California,

tAlfred P. Sloan Fellow and NSF Presidential Young Investigator.

Advances in Chemical Physics, Volume LXXXI, Edited by I. Prigogine and Stuart A. Rice. ISBN 0-471-54570-8 $\quad$ (C) 1992 John Wiley \& Sons, Inc. 
VI. Summary

Acknowledgments

References

\section{INTRODUCTION}

A primary objective of experimental and theoretical investigations in chemical physics has been to gain a detailed understanding of the transition state region in a chemical reaction. The transition state region is the region of the reactive potential energy surface where chemical change occurs, and studies which probe this region offer the promise of qualitative improvements in our understanding of the microscopic forces that govern the course of a chemical reaction. Although much of the effort in this area has concentrated on the extraction of properties of the transition state region through scattering experiments, a parallel and considerably smaller effort has focused on the development of spectroscopic methods which can be applied directly to the transition state. These "transition state spectroscopy" experiments are complicated by the short-lived nature of the transition state $\left(10^{-15}-10^{-12} \mathrm{sec}\right)$. In addition, the transition state region must be accessed in a sufficiently well-defined manner so that one can observe meaningful structure associated with the transition state.

A number of frequency and time-resolved techniques have been applied to the transition state in recent years. These have been admirably reviewed by Brooks ${ }^{1}$ and Zewail. ${ }^{2}$ Zewail and co-workers ${ }^{3,4}$ have carried out a particularly noteworthy set of experiments in which chemical reactions and photodissociation are monitored in real time using femtosecond lasers. This chapter focusses on a complementary type of transition state spectroscopy experiment performed in our laboratory in which photodetachment of a stable negative ion is used to probe the transition state region in bimolecular chemical reactions.

The principle behind photodetachment-based transition state spectroscopy is that if the geometry of a stable anion is similar to that of the neutral transition state, then one can photodetach the anion and prepare the unstable transition state complex in a well-defined manner. The idea of using photodetachment to create an unstable neutral species has been in the literature for some time. In 1968 Golub and Steiner ${ }^{5}$ reported the total photodetachment cross section of the anion $\mathrm{OH}^{-}\left(\mathrm{H}_{2} \mathrm{O}\right)$; the resulting $\mathrm{OH} \cdot \mathrm{H}_{2} \mathrm{O}$ complex dissociates rapidly. More recently, Brauman and coworkers ${ }^{6}$ measured total cross sections for the photodetachment of a series of anions $\mathrm{ROHF}^{-}$in which the neutral ROHF complex dissociates to $\mathrm{HF}+\mathrm{RO}$.

To obtain more detailed information on the dissociating neutral com- 
plex, one can use techniques such as negative ion photoelectron spectroscopy ${ }^{7,8}$ and threshold photodetachment spectroscopy. ${ }^{9}$ These methods can yield vibrational structure associated with the transition state even if dissociation occurs in as little as $10^{-14} \mathrm{sec}$. These techniques can be applied to unimolecular decomposition, as in the photoelectron spectrum of $\mathrm{HCO}^{-}$obtained by Lineberger and co-workers, ${ }^{10}$ which shows resolved transitions to vibrationally excited levels of $\mathrm{HCO}$ which lie up to $4000 \mathrm{~cm}^{-1}$ above the $\mathrm{H}+\mathrm{CO}$ dissociation limit. One can also study unimolecular isomerization, as in Lineberger's photoelectron spectrum of the vinylidene anion $\mathrm{C}_{2} \mathrm{H}_{2}^{-} .{ }^{11}$ Although the neutral vinylidene radical rapidly isomerizes to acetylene, vibrational features attributed to the vinylidene structure are observed in the spectrum.

During the last few years, we have applied photodetachment spectroscopy to the study of transition states in bimolecular chemical reactions. We have studied several hydrogen transfer reactions $\mathrm{A}+\mathrm{HB} \rightarrow \mathrm{HA}+\mathrm{B}$, where $A$ and $B$ are atomic or polyatomic species, via photodetachment of the stable anion $\mathrm{AHB}^{-} .{ }^{12}$ In many cases, the $\mathrm{AHB}^{-}$anion is strongly hydrogen-bonded with a dissociation energy of $\sim 1 \mathrm{eV}$ or higher, and its overall size and geometry are such that one obtains good overlap with the neutral transition state region upon photodetachment. One then obtains resolved vibrational progressions which reveal the spectroscopy and dissociation dynamics of the neutral AHB complex, and from this one can learn about the features of the A + HB potential energy surface near the transition state.

One of the primary motivations for conducting these studies was that they appeared to hold high promise for experimentally observing reactive resonances. In the early $1970 \mathrm{~s}$, quantum mechanical scattering calculations on model collinear potential energy surfaces for the $\mathrm{H}+\mathrm{H}_{2}$ reaction by Truhlar and Kuppermann ${ }^{13}$ and $\mathrm{Wu}$ and Levine ${ }^{14}$ revealed pronounced oscillatory structure in the reaction probability as a function of translational energy. This structure was attributed ${ }^{15}$ to reactive resonances which occur because of quasi-bound states with high probability density in the transition state region. In the following years, resonances were found in collinear scattering calculations for the $\mathrm{F}+\mathrm{H}_{2}$ reaction. ${ }^{16-19}$ Especially sharp resonance structure due to long-lived states of the collision complex was predicted in calculations by Pollak ${ }^{20}$ and Bondi et al..$^{21}$ for heavy + light-heavy reactions such as $\mathrm{Cl}+\mathrm{HCl}$ or $\mathrm{I}+\mathrm{HI}$. This is the mass combination relevant to most of the experiments performed in our laboratory.

Clearly, the experimental observation of resonances would be an important step in understanding the vibrational structure of the transition state. ${ }^{22}$ However, while resonances have been shown to exist in scattering calculations on three-dimensional (rather than collinear) potential energy sur- 
faces, ${ }^{23}$ their experimental detection in a scattering experiment is complicated by the contribution of many partial waves to the total reaction cross-section. This has been predicted ${ }^{24,25}$ to cause substantial blurring of resonances in total cross-section measurements, such as those recently performed on the $\mathrm{H}+\mathrm{H}_{2}$ reaction. ${ }^{26}$ Differential cross-section measurements on the $\mathrm{F}+\mathrm{H}_{2}$ reaction ${ }^{27}$ show evidence for reactive resonances, but the ultimate interpretation of these results requires a more accurate potential energy surface than is currently available.

The photodetachment experiment offers an excellent opportunity for the observation of reactive resonances. Long-lived quasi-bound states on the $A+\mathrm{HB}$ potential energy surface should appear as sharp structure in the $\mathrm{AHB}^{-}$photoelectron spectrum, provided these quasi-bound states have good Franck-Condon overlap with the anion. In our experiments, the anions are produced in a free-jet expansion which should result in substantial rotational cooling. This means that, subsequent to photodetachment, the total angular momentum available to the reaction is much more restricted than in a reactive scattering study of the same system. Resonances should therefore be more pronounced in the photodetachment experiment. The results and analysis presented below will show that resonances are responsible for some, but not all, of the structure seen in the $\mathrm{AHB}^{-}$photodetachment spectra.

The organization of this chapter is as follows. In Section II the experimental methods used in this work are described. Section III presents a general discussion of the theoretical methods useful in analyzing these spectra. Section IV covers the symmetric $\mathrm{Br}+\mathrm{HBr}$ and $\mathrm{I}+\mathrm{HI}$ reactions, which are studied by photodetaching the bihalide anions $\mathrm{BrHBr}^{-}$and $\mathrm{IHI}^{-}$. Finally, several asymmetric hydrogen exchange reactions are discussed in Section V.

\section{EXPERIMENTAL METHODS}

The spectra shown below were obtained using two negative ion photodetachment methods: "fixed-frequency" photoelectron spectroscopy and threshold photodetachment spectroscopy. Both experiments involve the generation of an internally cold, mass-selected negative ion beam, but different photodetachment and electron detection schemes are employed. The two instruments are described briefly in this section; more detailed descriptions may be found elsewhere. ${ }^{9,28}$

Figure 1 illustrates the principles behind the two techniques. In photoelectron spectroscopy (Fig. 1a), the negative ions are photodetached with a fixed-frequency laser and the kinetic energy distribution of the ejected photoelectrons is measured. All energetically accessible levels of the neu- 
a) 'fixed-frequency' photoelectron spectroscopy

(8 meV resolution)

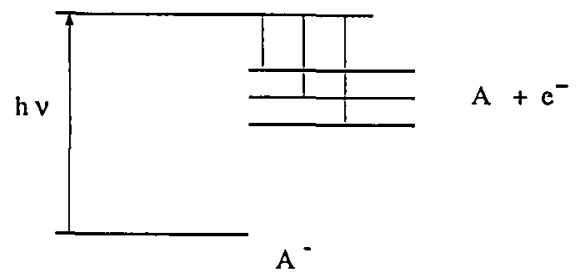

b) threshold photodetachment spectroscopy $(0.4 \mathrm{meV})$

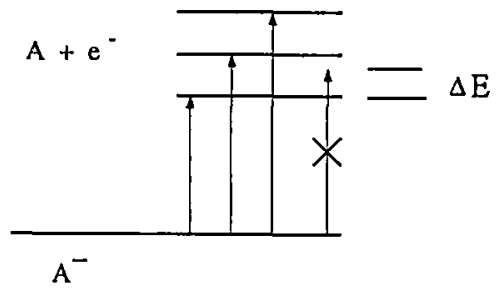

Figure 1. Energy levels and laser excitation scheme for (a) photoelectron spectroscopy and $(b)$ threshold photodetachment spectroscopy.

tral are populated according to their Franck-Condon overlap with the initial state of the anion. For a single photodetachment event, the electron kinetic energy (eKE) is given by

$$
\mathrm{eKE}=h \nu-E_{b}^{(-)}-E_{i}^{(0)}+E_{i}^{(-)}
$$

Here $h \nu$ is the photon energy and $E_{b}^{(-)}$is the energy difference between the anion and neutral ground states. In the case of $\mathrm{IHI}^{-}$photodetachment, where the IHI complex is unstable with respect to dissociation, $E_{b}^{(-)}$is the energy required to remove an electron from the ground state of $\mathrm{IHI}^{-}$ to form $\mathrm{I}+\mathrm{HI}(v=0) . E_{i}^{(-)}$and $E_{i}^{(0)}$ are the internal energies of the anion and neutral, respectively. For $\mathrm{IHI}^{-}$photodetachment, $E_{i}^{(0)}$ is measured relative to $\mathrm{I}+\mathrm{HI}(v=0)$.

Thus, the electron kinetic energy distribution exhibits peaks resulting from transitions between ion and neutral energy levels. In nearly all cases, the energy resolution of photoelectron spectroscopy is insufficient to discern rotational structure and one only learns about vibrational energy levels of the neutral and (occasionally) the anion. In our spectrometer, 


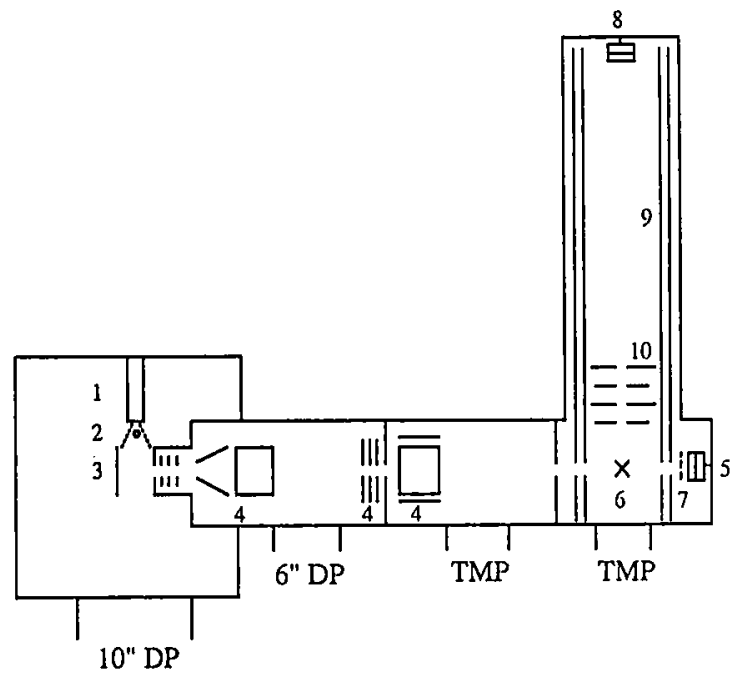

Figure 2. Schematic diagram of time-of-flight photoelectron spectrometer. Salient features are described in text (Ref. 28).

for example, the energy resolution of our time-of-flight analyzer is $8 \mathrm{meV}$ at $\mathrm{eKE}=0.65 \mathrm{eV}$ and degrades at higher electron kinetic energies as $(\mathrm{eKE})^{3 / 2}$. This is the instrumental resolution function used in the simulations of Sections IV and V.

Figure 2 shows a schematic of the negative ion photoelectron spectrometer used in these studies. ${ }^{28} \mathrm{~A}$ similar instrument is described by Posey et al. ${ }^{29}$ Because many of the $\mathrm{AHB}^{-}$anions of interest have high electron binding energies $\left(3.80 \mathrm{eV}\right.$ for $\mathrm{IHI}^{-}, 4.27 \mathrm{eV}$ for $\left.\mathrm{BrHBr}^{-}\right)$, a pulsed UV laser is the most appropriate photodetachment light source, and the entire instrument is based on pulsed technology. An excellent review of pulsed methods in ion spectroscopy is provided by Johnson and Lineberger, ${ }^{30}$ and several components of our instruments are described in more detail therein.

In the photoelectron spectrometer, negative ions are generated by expanding a mixture of neutral gases through a pulsed molecular beam valve (1) and crossing the molecular beam with a $1-\mathrm{keV}$ electron beam (2) just outside the valve orifice. The fast electrons produce relatively slow secondary electrons by ionization. These slow electrons, which rapidly thermalize due to the high gas density in front of the beam valve, efficiently produce negative ions through low-energy attachment processes. To make $\mathrm{BrHBr}^{-}$, for example, a $5 \% \mathrm{HBr} / \mathrm{Ar}$ mixture is used. The likely mechan- 
ism is formation of $\mathrm{Br}^{-}$via dissociative attachment to $\mathrm{HBr}$, and formation of $\mathrm{BrHBr}^{-}$via three-body clustering reactions:

$$
\mathrm{Br}^{-}+\mathrm{HBr}+\mathrm{M} \rightarrow \mathrm{BrHBr}^{-}+\mathrm{M}
$$

The ions are formed in the continuum flow region of the supersonic expansion and cool internally as the expansion progresses.

A time-of-flight mass spectrometer of the Wiley-Maclaren design ${ }^{31}$ is used to select the mass of the ion of interest. A small volume of negative ions is extracted at $90^{\circ}$ from the molecular beam by applying a negative pulse to the electrode (3). The ions then pass through a $1-\mathrm{kV}$ potential drop. As they traverse the $140-\mathrm{cm}$ drift region (4), the ions separate into bunches according to mass. The photodetachment laser pulse is timed so that it crosses the ion beam at (6) and interacts with the ion bunch of the desired mass. The spectra shown below were obtained with the fourth harmonic $(266 \mathrm{~nm}, 4.660 \mathrm{eV})$ or fifth harmonic $(213 \mathrm{~nm}, 5.825 \mathrm{eV})$ of a $\mathrm{Nd}$ :YAG laser. A small fraction $\left(10^{-4}\right)$ of the ejected photoelectrons is detected (8) at the end of a $100-\mathrm{cm}$ field free-flight tube (9) and energy analyzed by time of flight.

Considerably higher resolution can be obtained with threshold photodetachment spectroscopy. The principle of this method is shown in Fig. $1 b$. In this experiment, negative ions are photodetached with a tunable pulsed laser. At a given laser wavelength, only electrons produced with nearly zero kinetic energy are detected. The zero-kinetic energy spectrum plotted as a function of laser wavelength consists of a series of peaks, each corresponding to an ion $\rightarrow$ neutral transition. The width of the peaks is determined by the ability of the instrument to discriminate against photoelectrons produced with high kinetic energy. By adapting the methods developed by Müller-Dethlefs et al. for threshold photoionization of neutrals, ${ }^{32}$ we have achieved a resolution of $3 \mathrm{~cm}^{-1}(0.37 \mathrm{meV})$ with this instrument and were able to obtain a spectrum of $\mathrm{SH}^{-}$in which transitions between individual rotational levels of the anion and neutral were resolved. ${ }^{9}$

Figure 3 shows a schematic of the threshold photodetachment spectrometer. ${ }^{9}$ The ion source $(1,2)$ is similar to that shown in Fig. 2, but the ions pass through a skimmer and into a differentially pumped region prior to being accelerated to $1 \mathrm{keV}$. A coaxial time-of-flight mass spectrometer (3) of the design proposed by Bakker ${ }^{33}$ is used for mass separation. The anions are photodetached using an excimer-pumped dye laser with frequency-doubling capability.

In order to selectively detect zero-kinetic-energy photoelectrons, the region in which detachment occurs (4) is initially field-free. A weak extrac- 


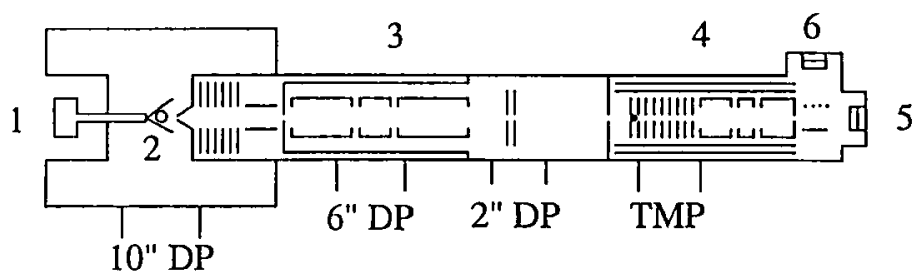

Figure 3. Schematic diagram of threshold photodetachment spectrometer: (1) pulsed beam valve, (2) electron beam, (3) coaxial time-of-flight mass spectrometer, (4) photodetachment region, (5) ion detector, (6) electron detector. Laser-ion beam interaction region is marked by (Ref. 9).

tion pulse is applied along the ion beam axis $200-300 \mathrm{~ns}$ after the photodetachment laser pulse. The purpose of this delay is to allow the higher energy electrons to spatially separate from the zero-kinetic-energy electrons. The higher-energy electrons that scatter perpendicularly to the ion beam axis are discriminated against since they will not pass through one of the many apertures between the detachment region and the electron detector (6). The higher-energy electrons that scatter along the beam axis will be in different regions of the extraction field when it is applied. They therefore emerge from the extraction region with different kinetic energies from the electrons produced with zero kinetic energy, arrive at the electron detector at different times, and are discriminated against by gated detection of the electron detector signal. The combination of spatial and temporal filtering is essential to achieving the ultimate resolution of this detection scheme.

\section{THEORETICAL METHODS}

In most negative-ion photodetachment experiments, a stable neutral species is formed by photodetaching a negative ion. Photoelectron spectra are typically analyzed by invoking the Born-Oppenheimer and Condon approximations. The electronic and vibrational degrees of freedom in the anion and neutral are assumed to be separable, so that the cross-section for photodetachment from anion vibrational level $v^{\prime \prime}$ to neutral vibrational level $v^{\prime}$ is given by

$$
\sigma\left(v^{\prime} \leftarrow v^{\prime \prime}\right) \propto\left|\tau_{e}\right|^{2}\left|\left\langle\psi_{v^{\prime}}^{(0)} \mid \psi_{v^{\prime \prime}}^{(-)}\right\rangle\right|^{2}
$$

Here $\tau_{\mathrm{e}}$ is the dipole matrix element between the electronic state of the anion and the (neutral $+\mathrm{e}^{-}$) continuum, which is assumed constant for a given electronic band. The approximations in Eq. (3) have been used 
in all theoretical treatments of photodetachment-based transition state spectroscopy experiments to date. In general, neither the anion or neutral potential energy surface is sufficiently well known to warrant a more sophisticated treatment.

The application of Eq. (3) to the spectra presented below is problematic since they all involve formation of an unbound, short-lived neutral complex. Thus, the vibrational wavefunction $\psi_{v^{\prime}}^{(0)}$ in Eq. (3) must be replaced by a scattering wavefunction. One can then simulate the photoelectron spectrum within a time-independent formalism, in which the FranckCondon overlap between the anion and neutral scattering wavefunctions is calculated as a function of energy. Alternatively, the spectrum can be simulated using time-dependent wavepacket analysis. Both types of simulations are discussed below.

\section{A. Time-Independent Analysis}

The time-independent analysis of these photodetachment experiments has been summarized nicely by Schatz. ${ }^{34}$ Equation (3) is replaced by

$$
\sigma(E) \propto\left|\left\langle\psi^{(0)}(E) \mid \psi_{v^{\prime \prime}}^{(-)}\right\rangle\right|^{2}
$$

where $\psi^{(0)}(E)$ is the scattering wavefunction supported by the neutral potential energy surface at energy $E$. In most scattering calculations, $E$ is measured relative to the neutral potential energy minimum in the asymptotic region for either reactants or products and differs from $E_{i}^{(0)}$ in Eq. (1) by the zero-point energy of the neutral fragments.

The accurate simulation of a photoelectron spectrum using Eq. (4) therefore requires the determination of $\mathrm{A}+\mathrm{HB}$ scattering wavefunctions over the energy range for which $\sigma(E)$ is nonnegligible. This is a formidable task which, so far, has only been attempted for triatomic systems, in which case a three-dimensional scattering wavefunction must be determined. Schatz has simulated the $\mathrm{ClHCl}^{-}$and $\mathrm{IHI}^{-}$photoelectron spectra in this manner ${ }^{35.36}$ using model London-Eyring-Polanyi-Sato (LEPS) potential energy surfaces for the $\mathrm{Cl}+\mathrm{HCl}$ and $\mathrm{I}+\mathrm{HI}$ reactions. The neutral wavefunctions are calculated with a coupled-channel method using hyperspherical coordinates. ${ }^{37}$ The calculations assume the anions to be groundstate harmonic oscillators and are restricted to total angular momentum $J=0$ for the anion and neutral. Both of these assumptions are justified by the low anion temperatures attained in the free-jet ion source, although the small rotational constants for $\mathrm{ClHCl}^{-}\left(B_{e}=0.09685 \mathrm{~cm}^{-1}\right)^{38}$ and $\mathrm{IHI}^{-}$ mean that several rotational levels will be populated even at $10 \mathrm{~K}$.

Using a different method for calculating the scattering wavefunctions, Zhang and Miller ${ }^{39}$ have performed a three-dimensional simulation of the 
$\mathrm{FH}_{2}^{-}$photoelectron spectrum, again assuming $J=0$. The calculation uses the $a b$ initio $\mathrm{F}+\mathrm{H}_{2}$ surface of Steckler et al. ${ }^{40}$ and the $a b$ initio anion geometry of Kendall et al. ${ }^{41}$ This simulation has recently been compared to the experimental $\mathrm{FH}_{2}^{-}$spectrum. ${ }^{42}$

A more approximate method for simulating $\mathrm{AHB}^{-}$photoelectron spectra in three dimensions has been developed by Gazdy and Bowman ${ }^{43}$ and used to simulate the $\mathrm{ClHCl}^{-}$and $\mathrm{IHI}^{-}$photoelectron spectra. They use an $L^{2}$ basis to obtain the neutral wavefunctions. This involves the imposition of boundary conditions which result in replacing three-dimensional continuum wavefunctions with bound-state wavefunctions. The $L^{2}$ method is well suited for determining the position of resonances, since the resonance wavefunctions are highly localized and are similar to bound-state wavefunctions. In regions of the $\mathrm{ClHCl}^{-}$and $\mathrm{IHI}^{-}$photoelectron spectra dominated by direct scattering, the $L^{2}$ method yields a many-line stick spectrum while the "exact" three-dimensional simulation ${ }^{35,36}$ typically yields a smaller number of peaks with varying widths. Nonetheless, the envelope of the $L^{2}$ stick spectrum matches the exact simulation reasonably well. The $L^{2}$ method is therefore a useful first test of a model threedimensional potential energy surface for a reaction of interest.

The simulation of $\mathrm{AHB}^{-}$photoelectron spectra is further simplified using Bowman's adiabatic bend approximation. ${ }^{44}$ This allows one to reduce the three-dimensional potential energy surface for a three-atom system to a two-dimensional surface by an approximate treatment of the bending motion. For example, the three-dimensional potential energy function for $\mathrm{Br}+\mathrm{HBr}$ is given by $V\left(Q_{1}, Q_{3}, \gamma\right)$, where

$$
\begin{aligned}
& Q_{1}=\frac{1}{\sqrt{2}}\left(r_{1}+r_{2}\right) \\
& Q_{3}=\frac{1}{\sqrt{2}}\left(r_{1}-r_{2}\right)
\end{aligned}
$$

are the symmetric and antisymmetric stretch symmetry coordinates $\left(r_{1}\right.$ and $r_{2}$ are the two $\mathrm{H}-\mathrm{Br}$ bond lengths) and $\gamma$ is the $\mathrm{Br}-\mathrm{H}-\mathrm{Br}$ bending angle. By assuming the bending motion is separable from the two stretches, $V\left(Q_{1}, Q_{3}, \gamma\right)$ is reduced to a two-dimensional "effective" collinear potential energy surface $V\left(Q_{1}, Q_{3}\right)$ via

$$
V\left(Q_{1}, Q_{3}\right)=V\left(Q_{1}, Q_{3}, \gamma=\pi\right)+\epsilon_{0}\left(Q_{1}, Q_{3}\right)
$$


Here $\epsilon_{0}\left(Q_{1}, Q_{3}\right)$ is the bending zero-point energy for the linear configuration of the nuclei specified by $Q_{1}$ and $Q_{3} . V\left(Q_{1}, Q_{3}\right)$ is therefore an approximate potential surface for the $\mathrm{BrHBr}$ complex in its ground-bending vibration. This approximation should be reasonably accurate if the reaction is collinearly dominated, so that photodetachment of a linear ion such as $\mathrm{BrHBr}^{-45,46}$ should not produce substantial bending excitation in the neutral complex.

Once the effective collinear surface is obtained, one can simulate the $\mathrm{AHB}^{-}$photoelectron spectrum via Eq. (4), where $\psi^{(0)}(E)$ is now a twodimensional scattering wavefunction. One can obtain $\psi^{(0)}(E)$ from an exact two-dimensional calculation,,$^{47}$ by using an $L^{2}$ basis,${ }^{48}$ or by making the DIVAH approximation ${ }^{49}$ in which $\psi^{(0)}(E)$ is assumed to be a product of two one-dimensional wavefunctions. The DIVAH approximation is quite accurate for heavy + light-heavy reactions ${ }^{21}$ and will be discussed in more detail in Section IV, where it is used to obtain an effective collinear surface for the $\mathrm{Br}+\mathrm{HBr}$ reaction. One can also perform an exact twodimensional simulation using the time-dependent methods described in the following section.

\section{B. Time-Dependent Analysis}

An alternative conceptual framework for analyzing and interpreting the photodetachment experiments is provided by time-dependent wavepacket analysis. This approach, initially developed by Heller, ${ }^{50}$ has been extended to a broad range of problems in spectroscopy and dynamics. ${ }^{51}$ Particularly relevant applications of this method to the material in this chapter include the work of Bisseling et al. ${ }^{52,53}$ who have used time-dependent analysis to study resonances in the $\mathrm{F}+\mathrm{DBr}$ reaction, and the work of Lorquet, ${ }^{54}$ who first used the time-dependent formalism to interpret the photoelectron spectrum of neutrals in which a dissociating ionic species is formed.

The basic idea behind the application of time-dependent analysis to photodetachment is as follows. In the time-independent analysis described above, the stationary state $\mathrm{A}+\mathrm{HB}$ scattering wavefunctions are calculated at many values of energy and the photoelectron spectrum is simulated via Eq. (4). The time-dependent perspective is based on the equivalent equation in which the photoelectron spectrum $\sigma(E)$ is expressed as the Fourier transform of a time-autocorrelation function $C(t)$,

$$
\sigma(E) \propto \int_{-\infty}^{\infty} \exp (i E t / \hbar) C(t) d t
$$


where

$$
C(t)=\langle\phi(t=0) \mid \phi(t)\rangle
$$

Here $\phi(t=0)$ is the initial wavepacket formed on the A + HB potential energy surface which, within the Condon approximation, is given by the stationary state wavefunction for the anion. The wavepacket $\phi(t)$ at later times is found by propagating $\phi(0)$ on the $\mathrm{A}+\mathrm{HB}$ potential energy surface,

$$
|\phi(t)\rangle=\exp [-i \hat{H} t / \hbar]|\phi(0)\rangle
$$

where $\hat{H}$ is the Hamiltonian on the neutral surface. In our implementation of time-dependent analysis, ${ }^{55}$ we restrict ourselves to two-dimensional wavepacket propagation on "effective" collinear potential energy surfaces. We use the propagation scheme of Kosloff and Kosloff ${ }^{56}$ in which the propagator is approximated by a second-order differencing scheme and the kinetic energy at each time $t$ is evaluated by the Fourier method. Although simulated photoelectron spectra using Eq. (6) are identical to those obtained using Eq. (4), the time-evolving wavepacket $\phi(t)$ and the autocorrelation function $C(t)$ provide additional insight concerning the $\mathrm{AHB}^{-}$photoelectron spectra. The connection between observed features in the spectra and dissociation dynamics of the AHB complex is particularly clear using the time-dependent formalism.

\section{SYMMETRIC HYDROGEN EXCHANGE REACTIONS}

\section{A. Photoelectron Spectrum of $\mathrm{BrHBr}$}

\section{Summary of Results}

The 213-nm photoelectron spectra ${ }^{28}$ of $\mathrm{BrHBr}^{-}$and $\mathrm{BrDBr}^{-}$(Fig. 4) show a series of well-resolved peaks with widely varying widths. The widths of the peaks labelled 0,2 , and 4 in the $\mathrm{BrHBr}^{-}$spectrum are 170, 80 , and $20 \mathrm{meV}$, respectively, and the widths of peaks $0,2,4$, and 6 in the $\mathrm{BrDBr}^{-}$spectrum are $175,100,64$, and $20 \mathrm{meV}$, respectively. The discussion in Sections IV.A.1-3 focusses on the peak positions and intensities. The peak widths will be discussed in Sections IV.A.4-6.

We first consider whether the peaks in these spectra correspond to transitions to levels of the neutral $\mathrm{BrHBr}$ complex which are bound or unbound with respect to $\mathrm{Br}+\mathrm{HBr}(v=0)$. This can be determined with 


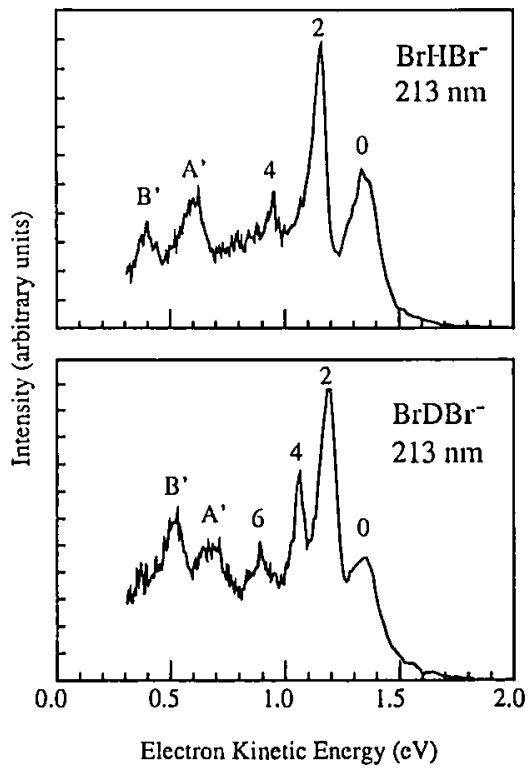

Figure 4. $\mathrm{BrHBr}^{-}$and $\mathrm{BrDBr}^{-}$photoelectron spectra taken at $213 \mathrm{~nm}$ photodetachment wavelength $(h \nu=5.825 \mathrm{eV})$. Peaks are labelled with $v_{3}^{\prime}$ quantum number (Ref. 28).

the energy level diagram, Fig. 5. This figure shows that peaks with electron kinetic energy less than $E_{0}^{\mathrm{ke}} \equiv \mathrm{h} \nu-D_{0}\left(\mathrm{BrHBr}^{-}\right)-\mathrm{EA}(\mathrm{Br})$ result from levels of the neutral complex that lie above $\mathrm{Br}+\mathrm{HBr}(v=0)$. Here, $\mathrm{h} \nu$ is the photon energy $(5.825 \mathrm{eV}), D_{0}\left(\mathrm{BrHBr}^{-}\right)$is the energy needed for the dissociation reaction $\mathrm{BrHBr}^{-} \rightarrow \mathrm{Br}^{-}+\mathrm{HBr}$, and $\mathrm{EA}(\mathrm{Br})$ is the electron affinity of $\mathrm{Br} . D_{0}\left(\mathrm{BrHBr}^{-}\right)$is given approximately by the enthalpy of dissociation of $\mathrm{BrHBr}^{-}, 0.91 \pm 0.05 \mathrm{eV},{ }^{57}$ and with $\mathrm{EA}(\mathrm{Br})=3.365 \mathrm{eV},{ }^{58}$ we find $E_{0}^{\mathrm{ke}}=1.55 \pm 0.05 \mathrm{eV}$. The peak at highest electron kinetic energy (Peak 0) appears at $1.353 \mathrm{eV}$ for $\mathrm{BrHBr}^{-}$, so all the observed peaks in the $\mathrm{BrHBr}^{-}$spectrum result from transitions to unbound states of the neutral complex.

We next consider the isotope effects observed in the spectra. Peak 0 occurs at the same electron kinetic energy in the $\mathrm{BrHBr}^{-}$and $\mathrm{BrDBr}^{-}$ spectra. The remaining peaks shift to higher electron energy, resulting in a smaller peak spacing. These two observations indicate that peak 0 is the origin of a vibrational progression in the neutral complex, and that the active mode involves $\mathrm{H}$ atom motion. None of the peaks appear to be "hot bands" originating from levels of the anion with excitation in the active mode, as these would shift to lower electron kinetic energy upon deuteration.

The active mode could be either the bend $\left(v_{2}\right)$ or antisymmetric stretch $\left(v_{3}\right)$ of the complex. $\mathrm{BrHBr}^{-}$is predicted to be linear, ${ }^{45,46}$ and, based on $a b$ initio surfaces calculated for the $\mathrm{Cl}+\mathrm{HCl}$ reaction, ${ }^{59,60}$ the minimum 


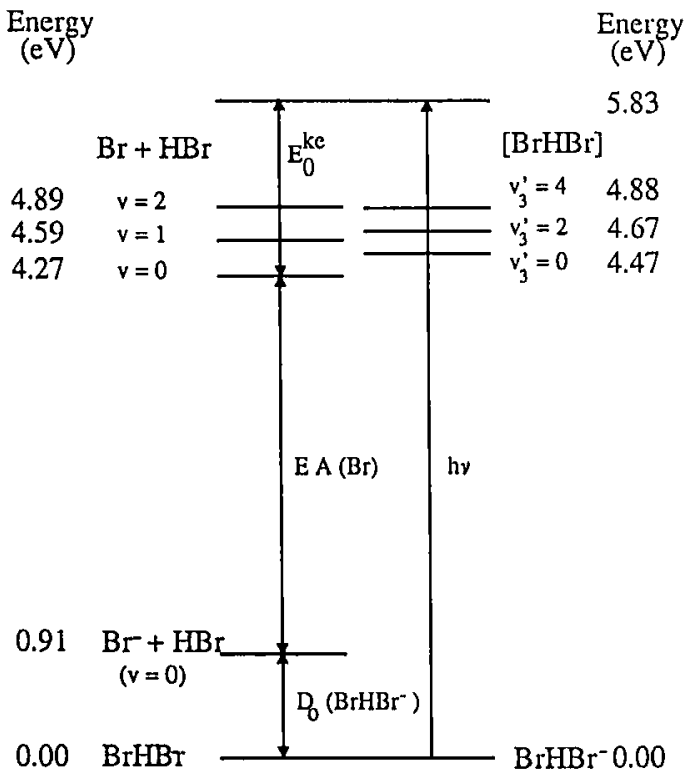

Figure 5. Energy level diagram for $\mathrm{BrHBr}^{-} / \mathrm{BrHBr}$ system (Ref. 28).

energy path for the $\mathrm{Br}+\mathrm{HBr}$ reaction is likely to be nearly collinear. An extended progression in the bend is therefore unlikely, and we assign the peaks to a progression in the $v_{3}$ mode. Symmetry considerations show that only transitions to even $v_{3}^{\prime}$ levels of the complex are allowed from the $v_{3}^{\prime \prime}=0$ level of the ion. The peaks in each spectrum are labelled by their $v_{3}^{\prime}$ quantum number. As discussed in more detail below, the two broad peaks at lowest energy in the $\mathrm{BrHBr}^{-}$and $\mathrm{BrDBr}^{-}$spectra $\left(\mathrm{A}^{\prime}\right.$ and $B^{\prime}$ ) do not appear to belong to the same progression as the higherenergy peaks and are assigned to transitions to an electronically excited $\mathrm{Br}+\mathrm{HBr}$ surface.

The energies of the $v_{3}^{\prime}$ states for the $\mathrm{BrHBr}$ complex and the $\mathrm{Br}+\mathrm{HBr}$ asymptotic vibrational energy levels are shown in Fig. 5. The vibrational spacing in the $\mathrm{BrHBr}$ complex is significantly smaller than that in free $\mathrm{HBr}$. For example, peaks 0 and 2 in the $\mathrm{BrHBr}^{-}$spectrum are separated by $0.194 \mathrm{eV}\left(1565 \mathrm{~cm}^{-1}\right)$, whereas the $v=0$ and $v=1$ levels of $\mathrm{HBr}$ are separated by $0.317 \mathrm{eV}\left(2557 \mathrm{~cm}^{-1}\right)$. The intuitive explanation for this is that the strong $\mathrm{HBr}$ bond in free $\mathrm{HBr}$ is replaced by two much weaker bonds in the $\mathrm{BrHBr}$ complex. This "red shift" indicates that we are probing the transition state region of the potential energy surface, where the hydrogen is interacting strongly with both bromine atoms. 


\section{Analysis: Preliminary Considerations}

The goal of the analysis of the $\mathrm{BrHBr}^{-}$and $\mathrm{BrDBr}^{-}$photoelectron spectra is to construct a potential energy surface for the $\mathrm{Br}+\mathrm{HBr}$ reaction which reproduces the experimental spectra in a simulation. This is accomplished using a potential energy surface with many adjustable parameters. By varying these parameters until the experimental spectra are reproduced, one can, in principle, map out the true potential energy surface in the region probed by photodetachment. Because of the large number of simulations required in this iterative procedure, the analysis was limited to constructing the best "effective" collinear surface (see Eq. (6)) that reproduced the experiments. This reduces the hardest part of the simulation, the calculation of the three-dimensional scattering wavefunctions in Eq. (4), to a much simpler two-dimensional problem.

In the past, LEPS potential energy surfaces have often been used to model the results from kinetics and reactive scattering experiments. However, while a LEPS surface can provide a qualitative framework for understanding a photoelectron spectrum of the type under consideration here, we have found that a more flexible function for the effective collinear surface is needed to reproduce the spectrum. The actual functional form used in our simulations is discussed in detail in Ref. 28.

The simulations also require wavefunctions for the anion. In contrast to $\mathrm{FHF}^{-61}$ and $\mathrm{ClHCl}^{-38}$ no gas-phase spectroscopic data exist for $\mathrm{BrHBr}^{-} . A b$ initio calculations by Peyerimhoff ${ }^{45}$ and Nomura ${ }^{46}$ predict a $D_{x h}$ equilibrium geometry for $\mathrm{BrHBr}^{-}$with an interhalogen distance, $R_{e}(\mathrm{BrBr})$, of 3.43 and $3.36 \AA$, respectively. Nomura predicts the $\nu_{1}$ frequency to be $200 \mathrm{~cm}^{-1}$ and the $\nu_{3}$ frequency to be $837 \mathrm{~cm}^{-1}$; the matrix isolation spectroscopy values for these frequencies are 164 and $728 \mathrm{~cm}^{-1}$, respectively. ${ }^{62}$ In our analysis, we assumed $R_{e}(\mathrm{BrBr})$ to be $3.50 \AA$. (Nomura's value became available subsequent to publication of our results.) We use harmonic oscillator wavefunctions with the matrix isolation values for the frequencies. Since all the peaks in the photoelectron spectra appear to originate from the ground state of the anion, this is assumed to be the initial anion vibrational state in all the simulations.

The end result of our analysis is the "best fit" effective collinear surface in Fig. 6. The method by which this surface is obtained is described in Section IV.A.4; for now, we assume it is the correct surface. The barrier height on this surface is $46.8 \mathrm{~kJ} / \mathrm{mol}$, and the saddle point occurs at $R_{\mathrm{Br}-\mathrm{Br}}=3.29 \AA$. This surface is plotted using modified hyperspherical coordinates $\rho$ and $z$, which, for the $\mathrm{Br}-\mathrm{H}-\mathrm{Br}$ mass combination are approximately proportional to the symmetry coordinates $Q_{1}$ and $Q_{3}$ (Eq. (5)): 


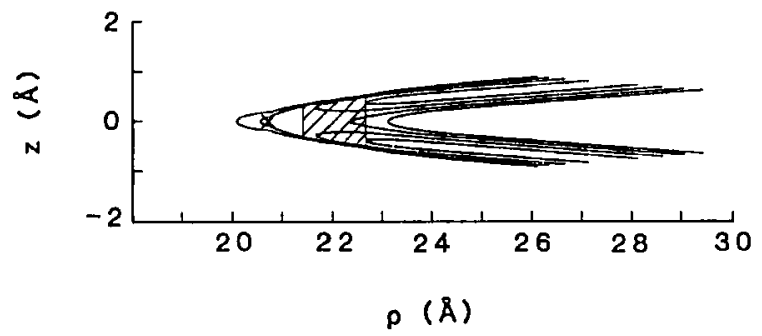

Figure 6. Contour plot of "best-fit" effective collinear potential energy surface for $\mathrm{Br}+\mathrm{HBr}$ reaction. Coordinates are defined in text (Eq. 10). The Franck-Condon region (see text) is shaded. Contours are at $-300,-325,-350$, and $-370 \mathrm{~kJ} / \mathrm{mol}$ with respect to three-atom dissociation (Ref. 28).

$$
\begin{gathered}
\rho=\sqrt{\frac{\mu_{\mathrm{Br}, \mathrm{HBr}}}{\mu_{\mathrm{HBr}}}} R_{\mathrm{Br}-\mathrm{Br}}=6.30 R_{\mathrm{Br}-\mathrm{Br}}=8.91 Q_{1} \\
z \approx \frac{Q_{3}}{\sqrt{2}}
\end{gathered}
$$

The approximations in Eq. (10) are valid for the heavy + light-heavy mass combination. The acute skew angle in Fig. 6 is characteristic of a heavy + light-heavy reaction such as $\mathrm{Br}+\mathrm{HBr}$ and is given by

$$
\phi_{m}=\tan ^{-1}\left\{\left[m_{\mathrm{H}}\left(m_{\mathrm{H}}+2 m_{\mathrm{Br}}\right) / m_{\mathrm{Br}}^{2}\right]^{1 / 2}\right\}=9.1^{\circ}
$$

The modified hyperspherical coordinates can be expressed in terms of the more familiar mass-scaled coordinates $x$ and $y$. The latter are defined for the reaction $\mathrm{Br}^{\prime}+\mathrm{HBr} \rightarrow \mathrm{HBr}^{\prime}+\mathrm{Br}$ by

$$
\begin{aligned}
& x=\sqrt{\frac{\mu_{\mathrm{Br}, \mathrm{HBr}}}{\mu_{\mathrm{HBr}}}} R_{\mathrm{Br}^{\prime}, \mathrm{HBr}} \\
& y=R_{\mathrm{H}-\mathrm{Br}}
\end{aligned}
$$

where $R_{\mathrm{Br}^{\prime}, \mathrm{HBr}}$ is the distance between the $\mathrm{Br}^{\prime}$ atom and the $\mathrm{HBr}$ center of mass. The two sets of coordinates are related by

$$
\begin{aligned}
& \rho=\left(x^{2}+y^{2}\right)^{1 / 2} \\
& z=\rho\left(\arctan \frac{y}{x}+\frac{\phi_{m}}{2}\right)
\end{aligned}
$$


The shaded region in Fig. 6 indicates the area on the neutral surface that is directly probed by our photodetachment experiment. The center of the shaded region, at $z=0, \rho=22.05 \AA$, corresponds to $R_{e}(\mathrm{BrBr})=$ $3.50 \AA$, the assumed interhalogen distance in $\mathrm{BrHBr}^{-}$. The extent of the shaded region along the $\rho$ coordinate represents the zero-point amplitude for the symmetric stretch in the ground vibrational state of the ion. Our spectra are very sensitive to the details of this Franck-Condon region of the potential energy surface, which lies quite close to the saddle point on the surface.

\section{One-Dimensional Simulation of $\mathrm{BrHBr}^{-}$Spectrum}

In Section IV.A.1, the first few peaks in the $\mathrm{BrHBr}^{-}$and $\mathrm{BrDBr}^{-}$spectra were assigned to a progression in the $v_{3}$ antisymmetric stretch mode of the neutral $\mathrm{BrHBr}$ complex on the basis of the observed isotope shift. This assignment is supported by a simple one-dimensional analysis based on the potential energy surface in Fig. 6. The $z$ coordinate in Fig. 6 is proportional to the antisymmetric stretch coordinate in the complex. By taking a cut along the $z$ axis through the center of the Franck-Condon region, one obtains an approximate antisymmetric stretch potential for the complex at the geometry probed by photodetachment. This is a double minimum potential with a barrier at $z=0$. We should therefore be able to simulate the peak positions and intensities in the photoelectron spectrum by calculating the Franck-Condon overlap between the anion $v_{3}^{\prime \prime}=0$ level and the eigenfunctions supported by a double minimum potential.

The harmonic antisymmetric stretch potential for $\mathrm{BrHBr}^{-}$is shown at the bottom of Fig. 7. The lower double minimum potential in Fig. 7 is the potential assumed for the $v_{3}$ mode in the ground electronic state of the neutral complex. ${ }^{63}$ The $v_{3}^{\prime \prime}=0$ levels for $\mathrm{BrHBr}^{-}$and $\mathrm{BrDBr}^{-}$are shown, as well as the first few eigenvalues for the $\mathrm{BrHBr}$ and $\mathrm{BrDBr}$ complexes. As discussed above, all the peaks in the spectra originate from the $v_{3}^{\prime \prime}=0$ anion level, and from this level, only transitions to even $v_{3}^{\prime}$ levels of the complex have nonzero intensity. The resulting one-dimensional simulations of the $\mathrm{BrHBr}^{-}$and $\mathrm{BrDBr}^{-}$spectra are superimposed on the experimental spectra. Reasonable agreement is obtained with the positions and intensities of the $v_{3}^{\prime}=0,2$, and 4 peaks in the $\mathrm{BrHBr}^{-}$ spectrum and the $v_{3}^{\prime}=0,2,4$, and 6 peaks in the $\mathrm{BrDBr}^{-}$spectrum. Note that the one-dimensional simulation yields a stick spectrum; to facilitate comparison with the experimental intensities, the sticks have been convoluted with Gaussians to match the experimental peak widths.

The two broad low-energy peaks $\left(A^{\prime}\right.$ and $\left.B^{\prime}\right)$ do not seem to belong to the same vibrational progression as the higher-energy peaks. It is likely 


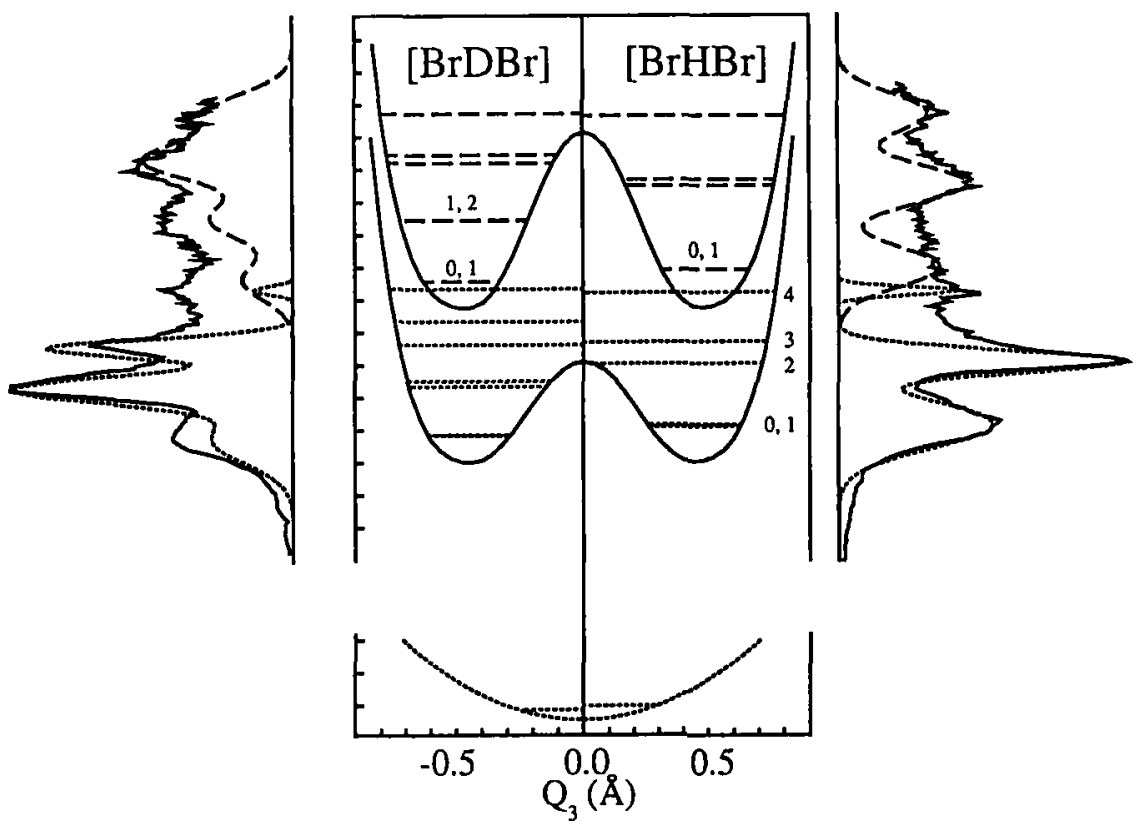

Figure 7. One-dimensional simulation of $\mathrm{BrHBr}^{-}$(right) and $\mathrm{BrDBr}^{-}$(left) photoelectron spectra. Anion $v_{3}$ harmonic oscillator potential and $v_{3}^{\prime \prime}=0$ levels of $\mathrm{BrHBr}^{-}$and $\mathrm{BrDBr}$ are shown at bottom. Double minimum $v_{3}$ potentials at middle and top are for ground and electronically excited neutral $\mathrm{Br} \mathrm{HBr}(\mathrm{BrDBr})$ complex, respectively. The first few $v_{3}$ energy levels are shown and $v_{3}$ quantum numbers are indicated for several levels including several nearly degenerate pairs. Results of one-dimensional simulations are compared with experimental spectra (solid) at the sides of the figure. For comparison with the experiment, the simulated peaks have been convoluted with Gaussians to match the experimental peak widths. Each tick mark on the vertical axis is $0.1 \mathrm{eV}$.

that peaks $A^{\prime}$ and $B^{\prime}$ are part of a second vibrational progression arising from a low-lying excited electronic state of the complex. The large isotope shift of peak $\mathrm{A}^{\prime}$ indicates that it is not the origin of this progression. However, the entire spectrum for each isotope can be simulated by assuming peaks $\mathrm{A}^{\prime}$ and $\mathrm{B}^{\prime}$ result from transitions to the $v_{3}^{\prime}=2$ and $v_{3}^{\prime}=4$ levels of the upper double-minimum potential shown in Fig. 7. The simulation from this potential is also shown in Fig. 7. The high barrier between the wells makes the $0 \leftarrow 0$ transition relatively weak.

The $v_{3}=0$ levels of the two double minimum potentials in Fig. 7 are separated by $0.49 \mathrm{eV}$. This is slightly larger than the ${ }^{2} \mathrm{P}_{1 / 2}-{ }^{2} \mathrm{P}_{3 / 2}$ spin-orbit splitting in $\mathrm{Br}(0.45 \mathrm{eV})$ and suggests that the upper potential corresponds to an electronically excited state of the complex which asymptotically correlates to $\mathrm{Br}{ }^{*}\left({ }^{2} \mathrm{P}_{1 / 2}\right)+\mathrm{HBr}$. Based on diatomics-in-molecules (DIM) 
calculations $^{64}$ on $\mathrm{F}+\mathrm{HF}$ and $\mathrm{Cl}+\mathrm{HCl}$, this excited state interaction is expected to be considerably more repulsive than the ground state. The high barrier between the wells of this potential is consistent with such a repulsive interaction.

The contribution of transitions to multiple potential energy surfaces in these spectra presents a complex and interesting problem. For example, the photoelectron spectrum of $\mathrm{ClHCl}^{-}$, which would at first seem like an ideal model system for study by this technique, shows barely resolved, very broad vibrational features. ${ }^{28.65}$ Recent $a b$ initio calculations by Yamashita and Morukuma ${ }^{66}$ suggest that the broad peaks result from overlapping transitions to both the $\mathrm{Cl}+\mathrm{HCl}$ ground state and a very low-lying excited state. We have also observed transitions to excited electronic states in the photoelectron spectrum of $\mathrm{IHI}^{-}$and several asymmetric bihalide anions. These are discussed in more detail below.

In summary, one can use the simple one-dimensional analysis scheme described above to obtain a good first-order understanding of the $\mathrm{BrHBr}^{-}$photoelectron spectrum. The analysis supports the assignment of the peaks in the spectrum to a progression in the $v_{3}$ antisymmetric stretch mode of the $\mathrm{BrHBr}$ complex. However, this is clearly only part of the story. The effective collinear surface in Fig. 6 has no wells in the transition state region, so one expects the $\mathrm{BrHBr}$ complex formed by photodetachment to dissociate rapidly. The dissociation dynamics of the complex affect the observed peak widths in the photoelectron spectrum, and this is not treated at all in our one-dimensional analysis.

The $v_{3}$ vibration treated in the one-dimensional analysis is largely decoupled from the dissociation coordinate of the $\mathrm{BrHBr}$ complex. This can be seen from Fig. 6; motion along the $z$ coordinate is nearly perpendicular to the minimum energy path that leads to reaction along the reactant and product valleys. To understand the dissociation dynamics of the $\mathrm{BrHBr}$ complex, we must at least include motion along the $\rho\left(v_{1}\right)$ coordinate on the $\mathrm{Br}+\mathrm{HBr}$ potential energy surface in our analysis. With reference to Fig. 6 again, the $\rho$ coordinate is clearly strongly coupled to dissociation. Thus, a two-dimensional analysis is necessary to incorporate dissociation of the complex.

\section{Two-Dimensional Simulation (Time-Independent)}

An exact two-dimensional simulation of the $\mathrm{BrHBr}^{-}$photoelectron spectrum can be generated by determining the Franck-Condon overlap between the ground state of the anion and the scattering wavefunctions supported by the effective collinear potential $V\left(Q_{1}, Q_{3}\right)$ or $V(\rho, z)$. In the $(\rho, z)$ coordinate system, the Schrodinger equation is 


$$
-\frac{1}{2 \mu_{\mathrm{HBr}}}\left\{\frac{\partial^{2}}{\partial \rho^{2}}+\frac{1}{\rho} \frac{\partial}{\partial \rho}+\frac{\partial^{2}}{\partial z^{2}}+V(\rho, z)\right\} \psi(\rho, z)=E \psi(\rho, z)
$$

Rather than solve the two-dimensional Schrödinger equation (9), we invoke an adiabatic approximation (the DIVAH approximation) that has been used by Römelt, Manz, and others ${ }^{21.49}$ in studies of heavy + lightheavy reactions. This approximation is based on the separation of time scales between the fast motion along the the $z$ coordinate, which primarily involves $\mathrm{H}$ atom motion, and the much slower motion along the $\rho$ coordinate which corresponds to displacement of the heavy halogen atoms.

The mathematics of this approximation are virtually identical to the Born-Oppenheimer approximation for diatomic molecules. The twodimensional scattering wavefunction $\psi(\rho, z)$ is assumed to be a product of two wavefunctions

$$
\psi(\rho, z)=\theta(z ; \rho) R(\rho)
$$

Here $\theta(z ; \rho)$ is the wavefunction for the antisymmetric stretch coordinate $z$. This function varies slowly with $\rho . R(\rho)$ is the wavefunction for motion along the $\rho$ coordinate. $\theta$ and $R$ satisfy the one-dimensional differential equations

$$
\left[-\frac{1}{2 \mu_{\mathrm{HBr}}} \frac{\partial^{2}}{\partial z^{2}}+V(\rho, z)\right] \theta(z ; \rho)=\epsilon_{\nu_{3}}(\rho) \theta(z ; \rho)
$$

and

$$
\left[-\frac{1}{2 \mu_{\mathrm{HBr}}} \frac{\partial^{2}}{\partial \rho^{2}}+U_{v 3}(\rho)\right] R(\rho)=E R(\rho)
$$

where

$$
U_{v_{3}}(\rho)=\epsilon_{v_{3}}(\rho)-\frac{1}{8 \mu_{\mathrm{HBr}} \rho^{2}}-\frac{1}{2 \mu_{\mathrm{HBr}}} Q_{v_{3} v_{3}}
$$

Equation (16) is the one-dimensional Schrodinger equation for a particle of mass $\mu_{\mathrm{HBr}}$ with potential energy $V(\rho, z)$ at a fixed value of $\rho$. (The appropriate reduced mass for the $\mathrm{Br}+\mathrm{DBr}$ reaction is $\left.\mu_{\mathrm{DBr}}\right)$. It is the same equation used in the one-dimensional analysis to determine the antisymmetric stretch energy levels and wavefunctions at the value of $\rho$ corresponding to the equilibrium geometry of $\mathrm{BrHBr}^{-}$. For each value of $\rho$ at which Eq. (16) is solved, one obtains a set of antisymmetric stretch 

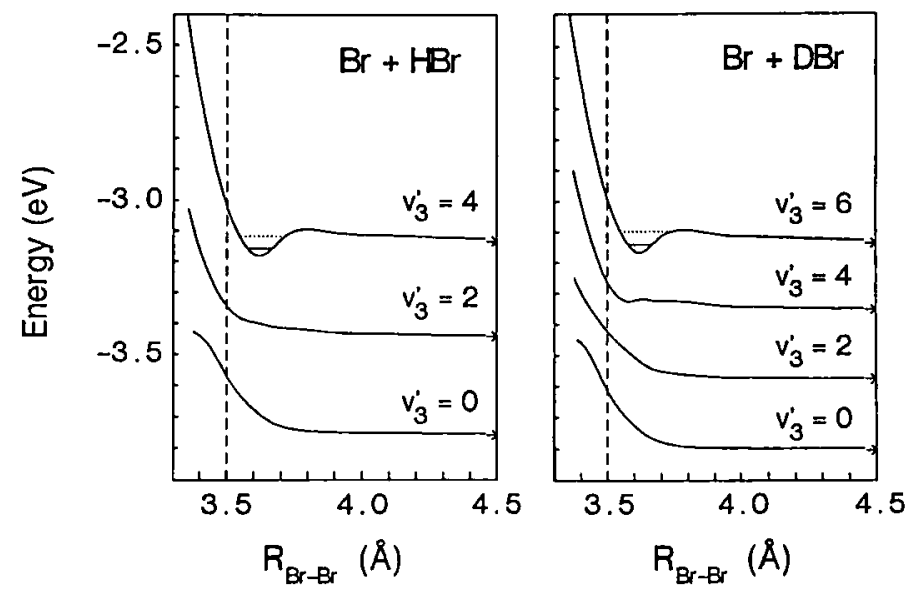

Figure 8. Effective potentials $U_{v_{3}}(\rho)(\mathrm{Eq} .18)$ for $\mathrm{Br}+\mathrm{HBr}$ and $\mathrm{Br}+\mathrm{DBr}$ derived from effective collinear surface in Fig. 6. Only potentials with even $v_{3}$ are shown (see text). Quasibound states are indicated by solid and dashed lines (solid lines denote states that can decay only by vibrational predissociation). The $\operatorname{Br}+\operatorname{HBr}(v=0,1,2)$ and $\mathrm{Br}+\mathrm{DBr}(v=0-3)$ asymptotic levels are indicated by arrows. The dashed vertical line at $R_{\mathrm{Br}-\mathrm{Br}}=3.50 \AA$ is the assumed value of $R_{r}(\mathrm{BrBr})$ in $\mathrm{BrHBr}^{-}$(Ref. 28).

eigenvalues $\epsilon_{v_{3}}(\rho)$ and eigenfunctions $\theta_{v_{3}}(z ; \rho)$. One therefore generates a set of "vibrationally adiabatic" curves $\epsilon_{v 3}(\rho)$ showing how the energy of the $v_{3}$ antisymmetric level varies with $\rho$, which is proportional to the interhalogen separation (Eq. (10)). In the limit of infinite $\rho$, the $v_{3}=2 m$ and $v_{3}=2 m+1$ levels become degenerate and correlate to the $\mathrm{Br}+\mathrm{HBr}$ $(v=m)$ vibrational energy level. The associated wavefunctions $\theta_{2 m}$ and $\theta_{2 m+1}$ have even and odd parity, respectively.

From the vibrationally adiabatic curves $\epsilon_{v_{3}}(\rho)$, one uses Eq. (18) to obtain the "effective potentials" $U_{v_{3}}(\rho)$. The DIVAH correction to $\epsilon_{v_{3}}(\rho), Q_{v_{3} v_{3}}$ in Eq. (18), is defined in Ref. 49. It is very small for a heavy + light-heavy reaction except near avoided crossings, and these do not occur for symmetric reactions. Figure 8 shows several of the lowestlying effective potentials $U_{v_{3}}(\rho)$ with even $v_{3}$ obtained from the potential energy surface in Fig. 6.

The significance of the effective potentials can be seen from Eq. (17), the one-dimensional Schrodinger equation for a particle with total energy $E$ and potential energy $U_{v_{3}}(\rho)$. For each value of $v_{3}, U_{v_{3}}(\rho)$ governs the motion of the $\mathrm{BrHBr}$ complex along the $\rho$ coordinate. The effective potentials $U_{0}(\rho)$ and $U_{2}(\rho)$ in Fig. 8 are purely repulsive; each set of the solutions $R_{0}(\rho)$ and $R_{2}(\rho)$ of Eq. (12) using these potentials is a continuum 
of one-dimensional scattering wavefunctions which vary smoothly as a function of $E$.

However, the potential $U_{4}(\rho)$ has a small well and can support states of the $\mathrm{BrHBr}$ complex which are quasi-bound along the $\rho$ coordinate in addition to a continuum of scattering wavefunctions; the energies of these quasi-bound states are indicated in Fig. 8. In a scattering calculation on this surface, these quasi-bound states would appear as resonances. Both states supported by the $U_{4}$ potential can decay by vibrational predissociation due to coupling with the repulsive effective potentials. In addition, the higher of the two quasi-bound states lies above the $\mathrm{Br}+\mathrm{HBr}(v=2)$ asymptote and can decay by tunneling through the barrier on the $U_{4}$ potential.

The effective potentials provide an inituitive explanation of the wide variation in peak widths observed in the $\mathrm{BrHBr}^{-}$and $\mathrm{BrDBr}^{-}$photoelectron spectra (Section IV.A.1). Since the $U_{0}$ and $U_{2}$ effective potentials are repulsive, transitions to the $v_{3}^{\prime}=0$ and $v_{3}^{\prime}=2$ levels of the complex should be quite broad. Specifically, the $v_{3}=0$ peak should be broader than the $v_{3}=2$ peak since the slope of the $U_{0}$ potential is steeper than that of the $U_{2}$ potential near $R_{\mathrm{Br}-\mathrm{Br}}=3.50 \AA$, the assumed interbromine distance in the anion. On the other hand, transitions to the quasi-bound states supported by the $U_{4}$ effective potential should yield narrow peaks in the photoelectron spectrum. The width of each peak is determined, in principle, by the lifetime of the quasi-bound state. For the $\mathrm{BrDBr}^{-}$ photoelectron spectrum, one expects the $v_{3}=0,2$, and 4 peaks to involve transitions to repulsive effective potentials, and the $v_{3}=6$ peak to result from a transition to a resonance supported by the $v_{3}=6$ effective potential. An examination of the effective potentials for $\mathrm{Br}+\mathrm{HBr}$ and $\mathrm{Br}+\mathrm{DBr}$ shows that as $v_{3}$ increases, the effective potentials become less repulsive and eventually develop wells. This general trend has been noted for several reactions.

We can now simulate the $\mathrm{BrHBr}^{-}$and $\mathrm{BrDBr}^{-}$photoelectron spectra by calculating the Franck-Condon overlap between the ground vibrational level of the anion, $\psi_{v_{1}^{(-)}=0}^{(-)}(\rho) \psi_{v_{3}^{(-)}=0}^{(-)}(z)$, and the neutral wavefunction in Eq. (10). The full spectrum is generated by summing over even values of $v_{3}^{\prime}$ :

$$
\sigma(E) \propto \sum\left|\int d \rho\left\{\int d z \theta_{v_{3}^{\prime}}(z ; \rho) \psi_{v_{3}^{\prime}=0}^{(-)}(z)\right\} R_{v_{3}^{\prime}}^{(E)}(\rho) \psi_{v_{1}^{\prime \prime}=0}^{(-)}(\rho)\right|^{2}
$$

Here $R_{v_{j}^{\prime}}^{(E)}(\rho)$ is the solution to Eq. (17) at total energy $E$. Note that the integral is zero for odd $v_{3}^{\prime}$ due to the opposite parities of the anion $\left(v_{3}^{\prime \prime}=\right.$ 


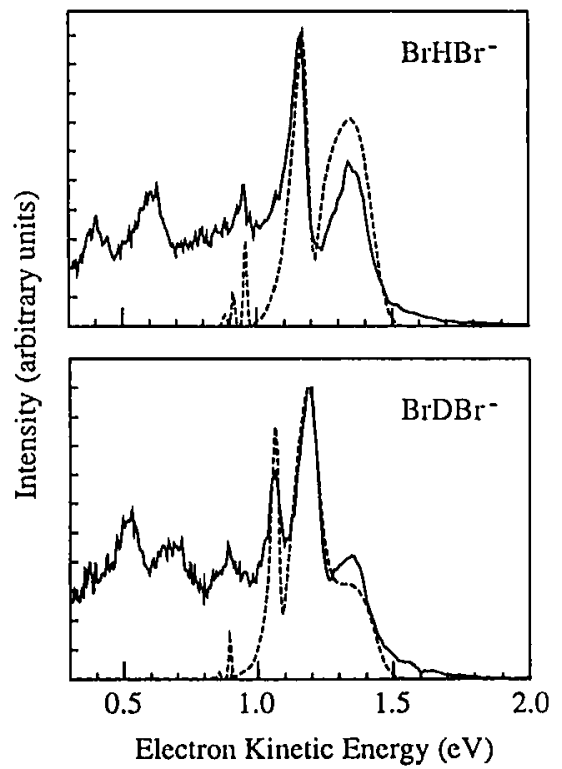

Figure 9. Results of two-dimensional simulation of $\mathrm{BrHBr}^{-}$and $\mathrm{BrDBr}^{-}$photoelectron spectra using $\mathrm{Br}+\mathrm{HBr}$ surface in Fig. 6. The simulated spectra (----) are convoluted with the instrumental resolution and are superimposed on the experimental spectra (Ref. 28).

0 ) and neutral (odd $v_{3}^{\prime}$ ) antisymmetric stretch wavefunctions. This is why only the effective potentials with even $v_{3}^{\prime}$ are shown in Fig. 8.

Figure 9 shows the simulated $\mathrm{BrHBr}^{-}$and $\mathrm{BrDBr}^{-}$spectra superimposed on the experimental spectra. The simulations are obtained by convoluting the results of Eq. (19) with the instrumental energy resolution. This has only a small effect on the broad peaks resulting from transitions to repulsive effective potentials, but has a large effect on transitions to the quasi-bound states. Within the DIVAH approximation, in which coupling between the effective potentials is neglected, quasi-bound states which can only decay by vibrational predissociation have infinite lifetimes; the peak widths of transitions to these states are determined entirely by the instrumental resolution. The comparison of the simulated and experimental spectra shows that the peak positions, intensities, and widths are within reasonable agreement for the ground state progression in both spectra.

\section{Time-Dependent Analysis of the $\mathrm{BrHBr}^{-}$Photoelectron Spectrum}

The discussion in the previous section focused on the relationship between the effective potentials in Fig. 8 and the features in the $\mathrm{BrHBr}^{-}$photoelectron spectrum. This was phrased in terms of Franck-Condon overlap between the anion ground state and the scattering wavefunctions supported by the $\mathrm{Br}+\mathrm{HBr}$ potential energy surface in Fig. 6 . In this section, a complementary analysis and simulation of the $\mathrm{BrHBr}^{-}$spectrum is 
presented in which an exact two-dimensional time-dependent wavepacket analysis is carried out on the same surface. This type of analysis provides an explicit connection between the spectrum and the dynamics of the $\mathrm{BrHBr}$ complex formed by photodetachment. The methods used in the simulation were outlined in Section III.2.

The analysis is carried out by allowing the initial wavepacket, $\phi(0)$, to evolve on the $\mathrm{Br}+\mathrm{HBr}$ potential energy surface. Within the FranckCondon approximation, $\phi(0)$ is obtained by projecting the anion groundstate wavefunction onto the neutral surface. This is illustrated in Fig. 10, $t=0$. The potential energy surface in Fig. 10 is the same as in Fig. 6, but is plotted using the mass-weighted coordinates $x$ and $y$ (Eq. 12). These coordinates are more convenient for wavepacket propagation than the hyperspherical coordinates discussed in the previous section. ${ }^{53}$ Note that $x$ and $y$ are nearly parallel to the $v_{1}$ and $v_{3}$ normal coordinates, respectively, in the anion.

The subsequent time evolution of this wavepacket is shown in Fig. 10. We observe rapid oscillation along the $y$ axis. In addition, after only $60 \mathrm{fs}$, most of the wavepacket bifurcates and begins moving down the reactant and product valleys. However, even after $300 \mathrm{fs,} \mathrm{a} \mathrm{small} \mathrm{fraction} \mathrm{of} \mathrm{the}$ original wavepacket remains in the transition state region. This remnant has four nodes along the $y$-coordinate and is due to quasi-bound states of the complex with $v_{3}=4$. These are the resonances which, in the timeindependent approach, occur because of the well in the $v_{3}=4$ effective potential (Fig. 8).

The time-dependent function $\phi(t)$ represents the evolution of $\phi(0)$, which is in turn a coherent superposition of scattering eigenfunctions $\psi_{\mathrm{E}}$ created at $t=0$. While $\phi(t)$ is uniquely related to the photoelectron spectrum via Eq. (8), it is important to emphasize that, in contrast to a shortpulse laser absorption experiment, photodetachment by a 10 -ns laser pulse does not actually create the localized wavepacket $\phi(0)$. Instead, each photodetachment event results in the formation of a well-defined neutral scattering state $\psi_{\mathrm{E}}$ with probability $\left|\left\langle\phi(0) \mid \psi_{\mathrm{E}}\right\rangle\right|^{2}$. Nonetheless, the plots of $\phi(t)$ are useful as they show what would occur if the initial wavepacket were created on the neutral potential energy surface; as such they give an overall picture of the dynamics of the $\mathrm{BrHBr}$ complex formed by photodetachment.

The time evolution of the initial wavepacket relevant to the photoelectron spectrum is described by the autocorrelation function $C(t)$ defined in Eq. (7). The modulus of this function, $|C(t)|$, which is plotted in Fig. 11, shows a rapidly decaying oscillation with a period of about $20 \mathrm{fs}$. This recurrence is due to the $v_{3}$ vibration of the complex, and the substantial decay of $C(t)$ after about $40 \mathrm{fs}$ represents movement of most of the 

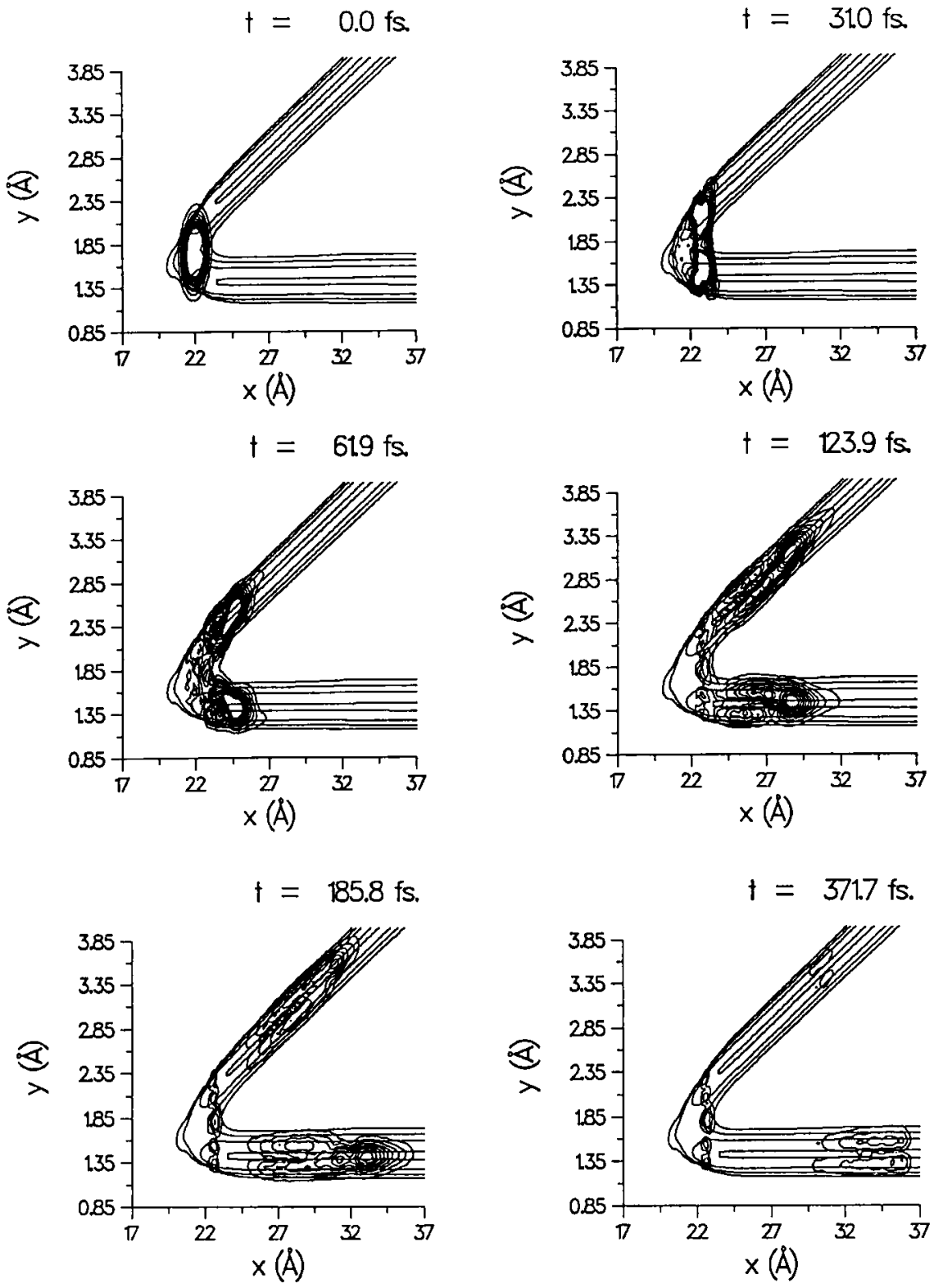

Figure 10. Time evolution of initially prepared wavepacket $\phi(0)$ on best-fit $\mathrm{Br}+\mathrm{HBr}$ surface. The surface and contours are the same as in Fig. 6 , but mass-scaled coordinates (Eq. 12) with an expanded $y$ axis are used. 
Figure 11. Modulus of time-autocorrelation function $|C(\ell)|$ (Eq. 8) obtained from wavepacket propagation on $\mathrm{Br}+\mathrm{HBr}$ surface in Fig. 6 .

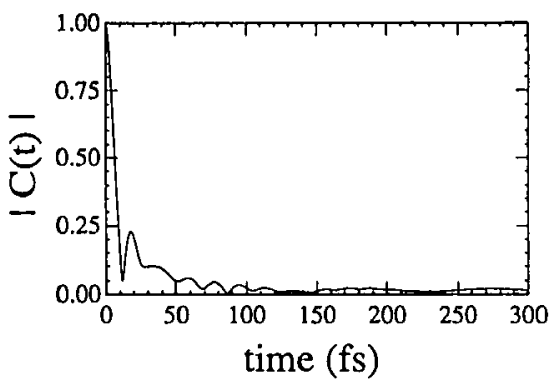

wavepacket out of the Franck-Condon region of the neutral potential energy surface. Roughly speaking, the time scale of this fast decay determines the width of the broad features in the photoelectron spectrum.

Thus, on this surface, the wavepacket dynamics primarily involve vibrational motion along the $v_{3}$ coordinate as the $\mathrm{BrHBr}$ complex rapidly dissociates. It is this vibrational motion occurring on the same (or slightly faster) time scale as dissociation that leads to resolved vibrational features in the photoelectron spectrum. For $t>120 \mathrm{fs}$, we observe a set of small, lower-frequency recurrences due to the resonances described above which are quasi-bound along the $v_{1}$ coordinate. The simulated photoelectron spectrum is obtained by taking the Fourier transform of $C(t)$ according to Eq. (8) and convoluting the result with the experimental resolution. This is therefore an exact two-dimensional simulation. One obtains the same simulation as with the time-independent method, confirming the suitability of the DIVAH approximation used in Section IV.A.4.

\section{Discussion of Results}

We now consider factors affecting the accuracy of our "best-fit" $\mathrm{Br}+\mathrm{HBr}$ potential energy surface. The first is the geometry of the $\mathrm{BrHBr}^{-}$anion. The effect of this can be understood with reference to the effective potentials in Fig. 8. The positions and widths of the peaks in the simulation corresponding to transitions to the repulsive $U_{0}$ and $U_{2}$ potentials depend strongly on the assumed value for $R_{e}(\mathrm{BrBr})$ in the anion. As mentioned previously, the most recent ab initio value ${ }^{46}$ for $R_{e}(\mathrm{BrBr})$ is $0.14 \AA$ smaller than that used in the simulations. Thus, our assumed value may well contribute to error in the best fit surface. In any case, an accurate experimental determination of the ion geometry would be of great assistance in these simulations. This could be obtained from the gas-phase vibrationrotation spectrum of $\mathrm{BrHBr}^{-}$measured by, for example, velocity-modulated infrared spectroscopy. ${ }^{67}$

The other vital issue in assessing the accuracy of our potential energy 
surface is the validity of the adiabatic bend approximation. Using this approximation, the surface must be quite repulsive along the minimum energy path in the Franck-Condon region in order to obtain satisfactory agreement with experiment, particularly with the widths of the peaks due to direct scattering. Such a surface yields steeply repulsive effective potentials for the lowest $v_{3}$ levels of the complex which are, in turn, necessary for the $v_{3}=0$ and 2 peaks in the simulated spectra to be sufficiently broad.

Figure $12 a$ shows an effective collinear LEPS surface which has the same barrier height and saddle-point geometry as our best-fit surface. The simulation of the photoelectron spectrum on this surface, shown in Fig. $12 b$, yields much narrower peaks because the slope of the minimum energy path is significantly less in the Franck-Condon region. In comparison with the best-fit surface, the effective potentials on the LEPS surface are less repulsive for low $v_{3}$ and the higher $v_{3}$ potentials exhibit deeper wells with more resonances.

Similarly, in the time-dependent picture, making the $\mathrm{Br}+\mathrm{HBr}$ surface repulsive in the Franck-Condon region insures that, on the best-fit surface, the initial wavepacket leaves this region rapidly and moves into the reactant and product valleys. This appears as a fast decay of the autocorrelation function $C(t)$, leading to broad peaks in the simulated photoelectron spectrum. Figure $12 c$ shows $|C(t)|$ resulting from wavepacket propagation on the LEPS surface in Fig. 12a. Clearly, the decay of $|C(t)|$ is much less rapid than in Fig. 11. In addition, at long times $(t>100 \mathrm{fs}$ ), Fig. $12 c$ shows the amplitude of the wavepacket in the FranckCondon region remains high, leading to pronounced resonance features in the simulated photoelectron spectrum.

The key question is whether the steeply repulsive nature of the bestfit surface in the Franck-Condon region is real or merely an artifact that results from simulating the spectrum within the adiabatic bend approximation. Schatz ${ }^{35}$ has simulated the $\mathrm{ClHCl}^{-}$spectrum in an exact threedimensional calculation using the LEPS surface of Bondi et al. ${ }^{21}$ for the $\mathrm{Cl}+\mathrm{HCl}$ reaction. In order to check the validity of the adiabatic bend approximation, we have compared his simulation to that obtained on the same model surface using this approximation. The results (after convolution with the experimental resolution) are similar. ${ }^{28}$ In particular, the widths of the $v_{3}=0$ peak, which is the only peak due to direct scattering, are quite close: $50 \mathrm{meV}$ in the three-dimensional simulation and $35 \mathrm{meV}$ in our simulation.

However, the extent of agreement between the two methods depends on the model potential energy surface used in the simulation. We will see in the next section that three-dimensional simulations of the $\mathrm{IHI}^{-}$photo- 


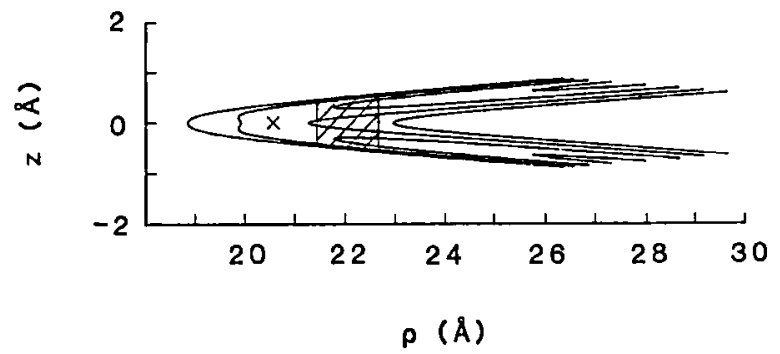

(a)

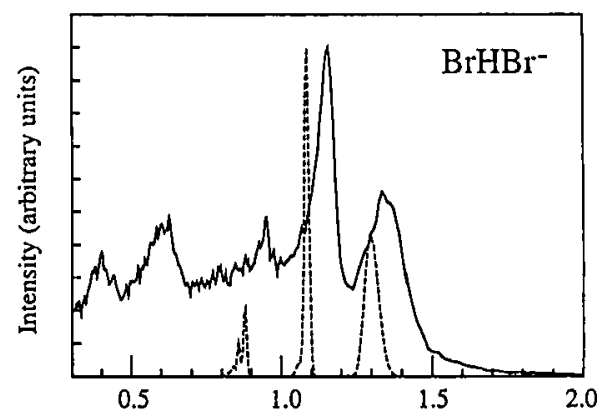

Electron Kinctic Energy (eV)

(b)

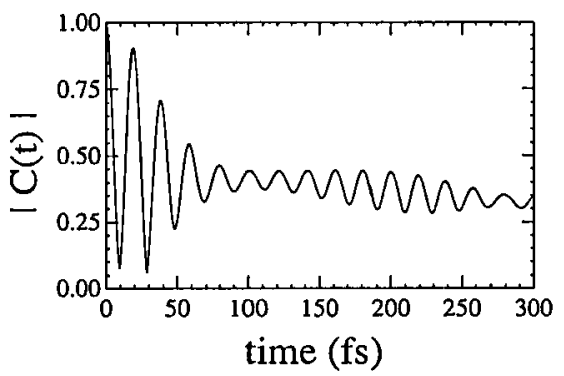

(c)

Figure 12. (a) Effective collinear $\mathrm{Br}+\mathrm{HBr}$ LEPS potential energy surface with same barrier height and saddle-point geometry as surface in Fig. 6 (Ref. 28). (b) Simulation of $\mathrm{BrHBr}^{-}$spectrum (-) using LEPS surface in (a) superimposed on experimental spectrum (solid) (Ref. 28). (c) $|C(t)|$ obtained from two-dimensional wavepacket propagation on LEPS surface in $(a)$.

electron spectrum $^{36}$ using the LEPS-A surface of Manz and Römelt ${ }^{68}$ for the $\mathrm{I}+\mathrm{HI}$ reaction show less agreement between the two methods. After describing the experimental and theoretical results for $\mathrm{IHI}^{-}$, we will return to the implications these have on our analysis of the $\mathrm{BrHBr}^{-}$spectrum. 


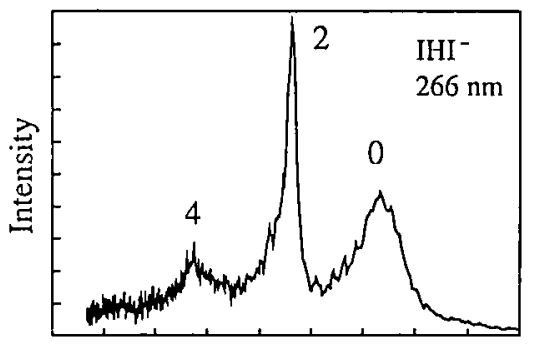

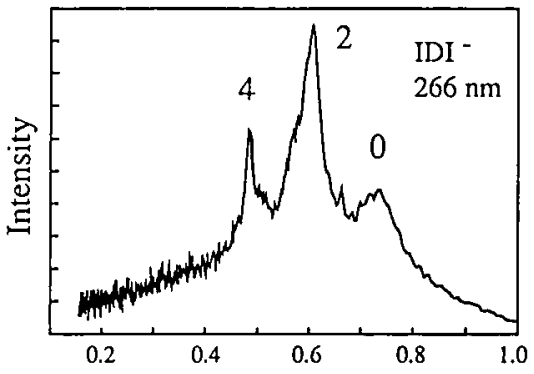

Electron Kinetic Energy (eV)
Figure 13. $\mathrm{IHI}^{-}$and $\mathrm{IDI}^{-}$photoelectron spectra taken at $266 \mathrm{~nm}(\mathrm{~h} \nu=4.660 \mathrm{eV})$. Peaks are labelled with $v_{3}^{\prime}$ quantum numbers (adapted from Ref, 69).

\section{B. Photoelectron and Threshold Photodetachment Spectra of IHI $^{-}$}

\section{Photoelectron Spectroscopy of $\mathrm{IHI}^{-}$and $\mathrm{IDI}^{-}$}

The photoelectron spectra of $\mathrm{IHI}^{-}$and $\mathrm{IDI}^{-}$at $266 \mathrm{~nm}$ are shown in Fig. $13 .{ }^{69}$ Each spectrum shows a progression of three clearly resolved peaks. Several similarities to the $\mathrm{BrHBr}^{-}$and $\mathrm{BrDBr}^{-}$spectra are evident. In the first place, the spectra show a large isotope shift; the peak spacing in the $\mathrm{IDI}^{-}$spectrum is clearly smaller than in the $\mathrm{IHI}^{-}$spectrum. The progressions are therefore assigned to the $v_{3}$ antisymmetric stretch of the neutral IHI complex. As in the $\mathrm{BrHBr}^{-}$spectra, only transitions to even $v_{3}$ levels of the complex occur. For each peak, the $v_{3}$ quantum number in the neutral complex is indicated in Fig. 13. Secondly, the frequency of the $v_{3}$ mode in the complex is substantially less than in free HI. The spacings between peaks 0 and 2 in the $\mathrm{IHI}^{-}$and $\mathrm{IDI}^{-}$spectra are $0.169 \pm 0.012 \mathrm{eV} \quad\left(1360 \pm 100 \mathrm{~cm}^{-1}\right)$ and $0.126 \pm 0.012 \mathrm{eV} \quad(1020 \pm$ $100 \mathrm{~cm}^{-1}$ ), respectively. The vibrational frequencies in HI and DI are 2309 and $1633 \mathrm{~cm}^{-1}$, respectively. The lower frequency in the complex is attributed to the weakening of the $\mathrm{HI}$ bond near the transition state of the $\mathrm{I}+\mathrm{HI}$ reaction. Finally, we note that, just as in the $\mathrm{BrHBr}^{-}$spectra, the peak widths in the $\mathrm{IHI}^{-}$and $\mathrm{IDI}^{-}$spectra show considerable variation. The $v_{3}^{\prime}=4$ peak in the $\mathrm{IDI}^{-}$spectrum is only $0.013 \mathrm{eV}$ wide. The peak widths in these spectra will be discussed in more detail below. 
Figure 14. $\mathrm{IHI}^{-}$and $\mathrm{IDI}^{-}$photoelectron spectra taken at $213 \mathrm{~nm}$.
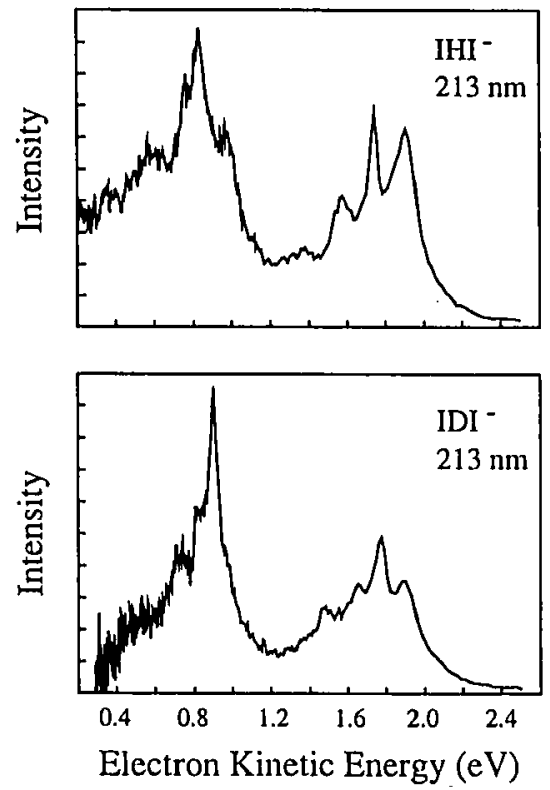

All the peaks in both spectra occur at electron kinetic energies less than $E_{0}^{\mathrm{ke}}=0.86 \pm 0.13 \mathrm{eV}$, the energy corresponding to formation of $\mathrm{I}+\mathrm{HI}(v=0, j=0)$. (The error bars are entirely due to the uncertainty in the enthalpy of dissociation for $\mathrm{IHI}^{-}: 0.74 \pm 0.13 \mathrm{eV} .{ }^{57}$ ) This means all the peaks are due to transitions to states of the IHI complex with sufficient energy to dissociate. Manz, Pollak, and co-workers ${ }^{70}$ and Clary and Connor ${ }^{71}$ have predicted the existence of "vibrationally bound" levels of IHI which actually lie below $\mathrm{I}+\mathrm{HI}(v=0, j=0)$, based on the LEPS-A surface ${ }^{68}$ proposed for the I + HI reaction. Our spectra show no evidence for truly bound IHI states; the discrepancy between experiment and theory is most likely due to inaccuracies in the LEPS surface used in the calculations.

Figure 14 shows the $\mathrm{IHI}^{-}$and $\mathrm{IDI}^{-}$photoelectron spectra taken at $213 \mathrm{~nm}$. The progressions at the $266 \mathrm{~nm}$ spectrum appear in Fig. 14 at higher electron kinetic energy (and lower resolution). In the 213-nm spectrum, these progressions include a fourth, small peak at lower electron energy ( $1.38 \mathrm{eV}$ in the $\mathrm{IHI}^{-}$spectrum, $1.47 \mathrm{eV}$ in the $\mathrm{IDI}^{-}$spectrum); at $266 \mathrm{~nm}$, the electrons corresponding to this peak are too low in energy to pass through our time-of-flight analyzer. The most prominent feature in the 213-nm spectra is the appearance of a second, intense band. In the $\mathrm{IHI}^{-}$spectrum, the separation between the origins of the two bands is 
$0.94 \mathrm{eV}$, which is essentially identical to the spin-orbit splitting in I. It therefore appears that we are observing transitions to the ground state I + HI surface and an excited-state surface that asymptotically correlates to $\mathrm{HI}+\mathrm{I}^{*}\left({ }^{2} \mathrm{P}_{1 / 2}\right)$. The two bands are better separated than the corresponding bands in the $\mathrm{BrHBr}^{-}$spectrum, which is expected since the spin-orbit splitting in I is about twice that in $\mathrm{Br}$.

The ground state $\mathrm{IHI}^{-}$photoelectron spectrum has been simulated by our group using the methods described in Sections IV.A.4-5. In addition, three-dimensional simulations (with $J=0$ ) have been performed by Gazdy and Bowman, ${ }^{43}$ using an $L^{2}$ basis for the I $+\mathrm{HI}$ scattering wavefunctions, and by Schatz, ${ }^{36}$ using exact $\mathrm{I}+\mathrm{HI}$ scattering wavefunctions. The simulated spectra from all three methods are shown in Fig. 15(a)-(c). All the simulations assume $\mathrm{IHI}^{-}$to be linear and centrosymmetric with an equilibrium interiodine distance $R_{e}=3.88 \AA$ and use the LEPS-A potential energy surface for the $\mathrm{I}+\mathrm{HI}$ reaction. In comparison to our best-fit surface for the $\mathrm{Br}+\mathrm{HBr}$ reaction, the LEPS-A surface for $\mathrm{I}+\mathrm{HI}$ has a considerably smaller barrier $(4.8 \mathrm{~kJ} / \mathrm{mol})$ and is much less repulsive along the minimum energy path in the Franck-Condon region. If we calculate the effective potentials on the LEPS-A surface using the adiabatic bend approximation, we find that they have deeper wells and support more resonances than the analogous curves in Fig. 8. Thus, one might expect a considerable amount of resonance structure in simulations of the $\mathrm{IHI}^{-}$ spectrum.

Indeed, all the simulations show that the $v_{3}^{\prime}=2$ feature in the $\mathrm{IHI}^{-}$ spectrum appears not as a single broad peak, but rather as a progression of peaks spaced by about $12 \mathrm{meV}\left(100 \mathrm{~cm}^{-1}\right)$. The closely spaced peaks represent transitions to quasi-bound symmetric stretch levels of the complex. These levels are analogous to those discussed in the $\mathrm{Br}+\mathrm{HBr}$ analysis and are responsible for resonances in the I + HI reaction. The simulation by Schatz in Fig. 15c, which is the most realistic, shows three closely spaced peaks associated with the $v_{3}^{\prime}=2$ level of [IHI] resulting from transitions to the $v_{1}^{\prime}=0,1$, and 2 symmetric stretch levels of the complex. The linewidths of these peaks are $2-5 \mathrm{meV}$, indicating resonance lifetimes of several hundred femtoseconds. Our simulation as well as that of Gazdy and Bowman (Fig. 15b) also predict resonance structure underlying the $v_{3}^{\prime}=4$ peak in the $\mathrm{IHI}^{-}$spectrum. Resonances are also observed in simulations of the IDI ${ }^{-}$spectrum. ${ }^{34,69}$

On the other hand, the simulations show that the $v_{3}^{\prime}=0$ feature in the $\mathrm{IHI}^{-}$spectrum is dominated by overlap with direct scattering wavefunctions rather than transitions to quasi-bound IHI states. Nonetheless, this feature is structured even in Schatz's "exact" simulation, where the 


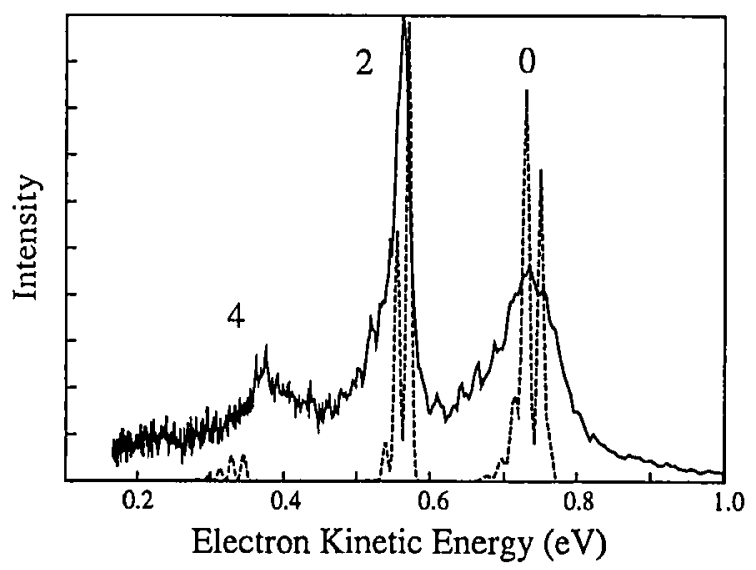

(a)

Figure 15. (a)

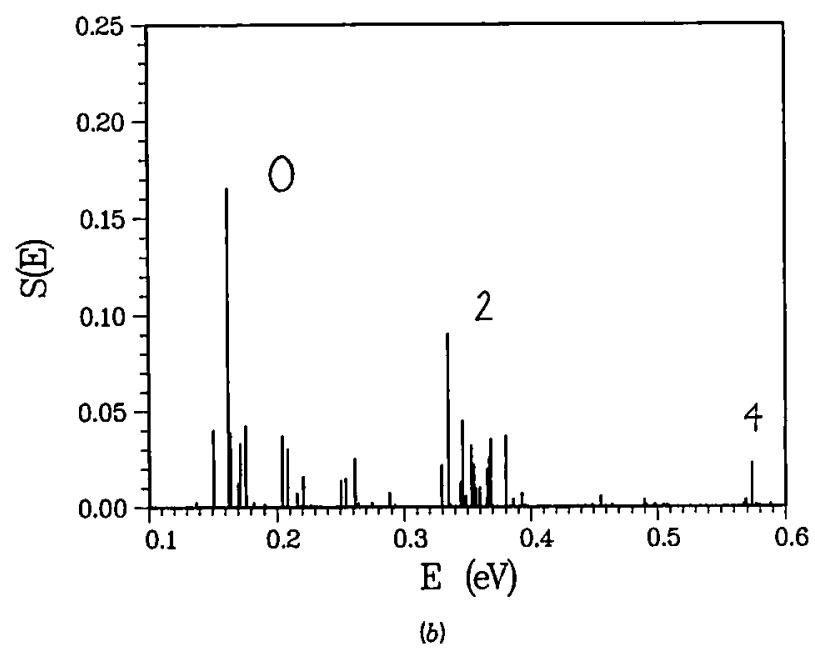

Figure 15. (b).

$v_{3}^{\prime}=0$ feature consists of a series of peaks spanning $0.1 \mathrm{eV}$, rather than the single broad peak in the experimental spectrum. The origin of this structure is discussed in Section IV.B.2.

To summarize, the simulated $\mathrm{IHI}^{-}$spectrum shows considerably more structure than the experimental spectrum. However, the LEPS-A surface used in these simulations is approximate at best. Thus, one must ask if the resonance and structured direct-scattering features in the simulations 


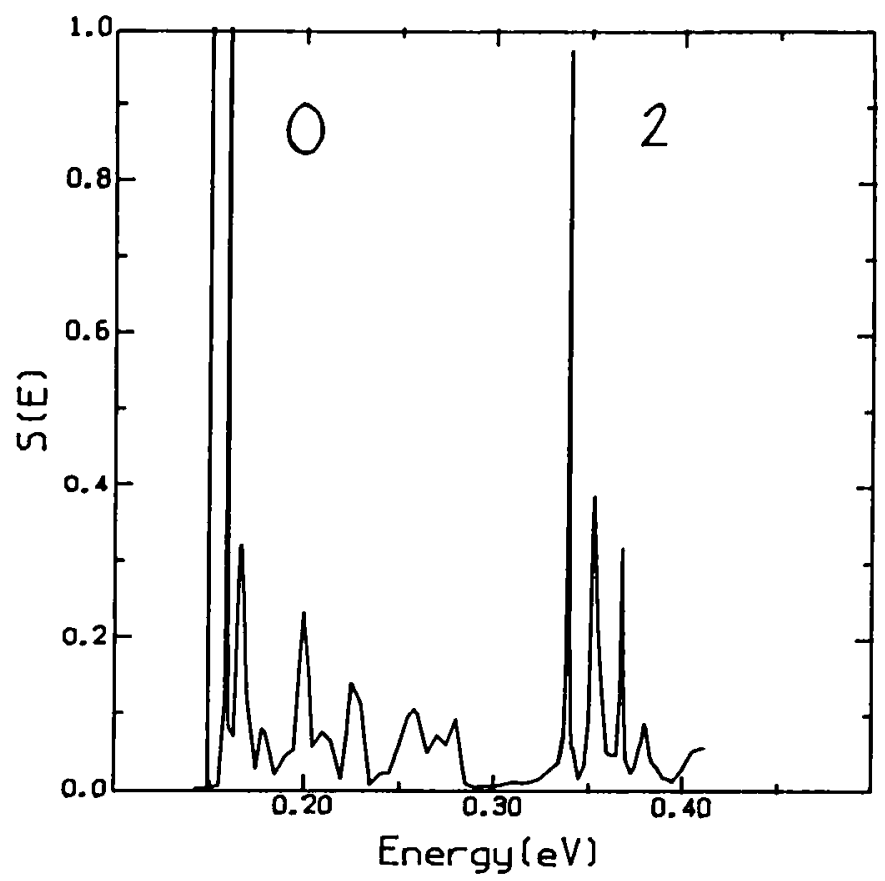

(c)

Figure 15. (a) Simulation of $\mathrm{IHI}^{-}$photoelectron spectrum using effective collinear LEPS-A I + HI surface from Ref. 68. The simulation (- $\longrightarrow$ is superimposed on the 266$\mathrm{nm}$ experimental spectrum. The simulated spectrum is shifted down by $0.100 \mathrm{eV}$ to match the experimental spectrum. (b) Three-dimensional $L^{2}$ simulation of $\mathrm{IHI}^{-}$spectrum (Ref. 43). (c) Exact three-dimensional simulation of $\mathrm{IHI}^{-}$spectrum (Ref. 36). Note that different energy scales are used in the simulations. In $(b)$ and $(c), E$ is measured relative to the bottom of the HI well in the asymptotic region of the LEPS-A surface. $E$ and eKE ( $a$ ) are related by $E=1.00-\mathrm{eKE}$, assuming that $E_{b}^{(-)}=3.80 \mathrm{eV}$ for $\mathrm{IHI}^{-}$(Eq. 1).

are real and are not seen in the $\mathrm{IHI}^{-}$photoelectron spectrum due to the limited resolution of the experiment, or whether the simulated features are artifacts resulting from inaccuracies in the LEPS-A surface. In order to answer this question, higher-resolution studies of $\mathrm{IHI}^{-}$were performed using our threshold photodetachment spectrometer. These experiments are described in the next section.

\section{Threshold Photodetachment Spectroscopy of $\mathrm{IHI}^{-}$}

The threshold photodetachment spectra of the $v_{3}^{\prime}=2,4$, and 0 peaks are shown in Figs. 16-18. ${ }^{72}$ The horizontal axis at the bottom of each plot shows the photodetachment wavelength $\lambda$. For convenient comparison with Fig. 13, the top axis shows the corresponding electron kinetic energy 


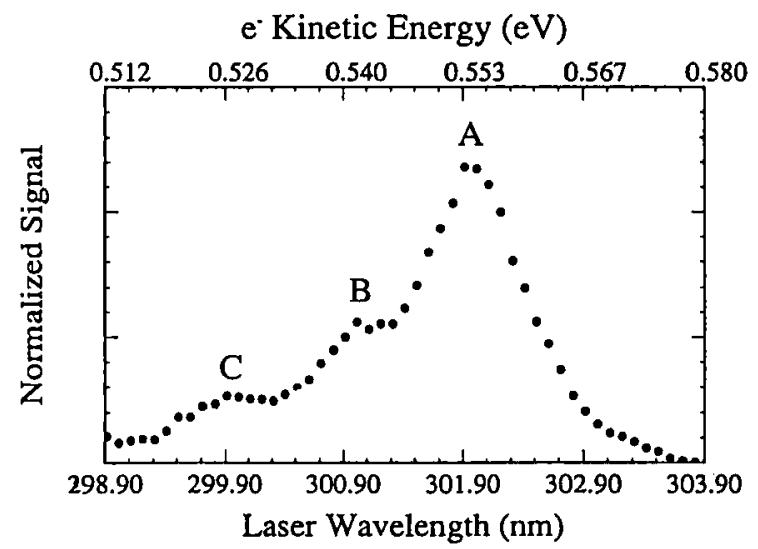

Figure 16. Threshold photodetachment spectrum of $v_{3}^{\prime}=2$ peak in $\mathrm{IHI}^{-}$photoelectron spectrum (see Fig. 13). The points are spaced by $0.1 \mathrm{~nm}\left(\sim 10 \mathrm{~cm}^{-1}\right)$ (Ref. 72$)$.

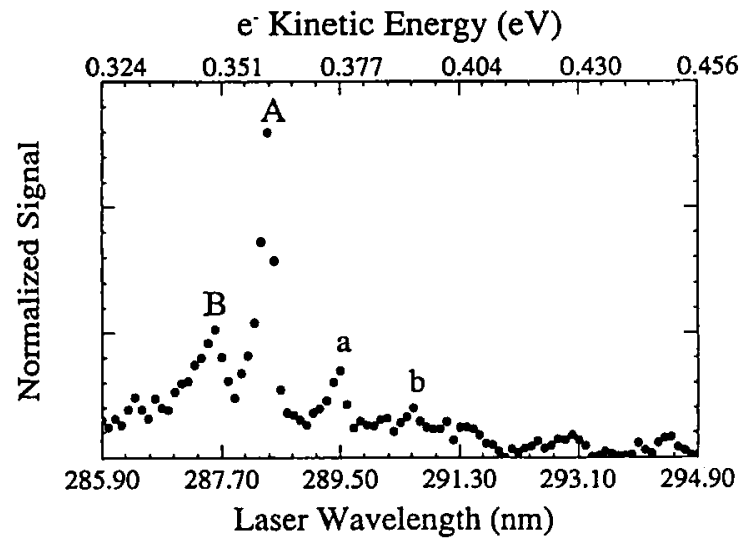

Figure 17. Threshold photodetachment spectrum of $v_{3}^{\prime}=4$ peak in $\mathrm{IHI}^{-}$photoelectron spectrum. Point spacing is $0.1 \mathrm{~nm}$ (Ref. 72).

(eKE) that would result from a fixed-frequency photoelectron spectrum at $266 \mathrm{~nm}$; the two axes are related by eKE $=1240(1 / 266-1 / \lambda)$.

The threshold photodetachment spectrum of the $v_{3}^{\prime}=2$ peak (Fig. 16) reveals three partially resolved peaks spaced by approximately $12 \mathrm{meV}$. The peak widths are about $12 \mathrm{meV}$, and the intensity of the peaks decreases towards shorter wavelength. The experimental peak spacings and intensities are quite similar to the simulations of the $v_{3}^{\prime}=2$ feature in Fig. 15. The major difference is that in the simulation by Schatz, ${ }^{36}$ the peaks in the symmetric stretch progression are narrower $(2 \mathrm{meV})$ and are there- 
fore fully resolved. Nonetheless, the correspondence between the experimental and simulated spectra strongly suggests we are seeing a progression in quasi-bound IHI symmetric stretch states.

Figure 17 shows the threshold photodetachment spectrum of the $v_{3}^{\prime}=$ 4 feature in the $\mathrm{IHI}^{-}$photoelectron spectrum. Four well-resolved peaks are evident in this spectrum. Peaks $\mathrm{A}$, $\mathrm{a}$, and $\mathrm{b}$ are evenly spaced by an interval of $16.0 \mathrm{meV}\left(129 \mathrm{~cm}^{-1}\right)$, while peaks $A$ and $B$ are separated by $12.5 \mathrm{meV}\left(101 \mathrm{~cm}^{-1}\right)$. The peaks are substantially narrower than those in Fig. 16; peaks A and a are $3.7 \mathrm{meV}$ wide, and peak $B$ is $5.6 \mathrm{meV}$ wide.

The presence of two intervals among the peaks in Fig. 17 suggests the presence of two progressions. Matrix isolation studies ${ }^{12}$ on $\mathrm{IHI}^{-}$have yielded a value of $129 \mathrm{~cm}^{-1}$ for the symmetric stretch frequency in the ion, in excellent agreement with the spacing between peaks $A$, a, and $b$. We therefore assign peaks $a$ and $b$ to hot-band transitions originating from the $v_{1}^{\prime \prime}=1$ and $v_{1}^{\prime \prime}=2$ symmetric stretch levels of the ion and terminating in the same level of the neutral as peak $A$. The a/A intensity ratio yields a vibrational temperature of $100 \mathrm{~K}$ for the $\mathrm{IHI}^{-}$ions.

Peaks $\mathrm{A}$ and $\mathrm{B}$ are assigned to transitions between the $v_{1}^{\prime \prime}=0$ level of the ion and two different symmetric stretch levels of the IHI complex. This assignment is supported by the peak spacing of $101 \mathrm{~cm}^{-1}$, and with the noticeably different peak widths which imply that the transitions are to two distinct levels of the complex with different lifetimes. The widths yield lower bounds of 180 and $120 \mathrm{fs}$ for the upper-state lifetimes of $\mathrm{A}$ and $B$, respectively. Since the symmetric stretch vibration of $[\mathrm{IHI}]$ is strongly coupled to the dissociation coordinate of the complex, it seems

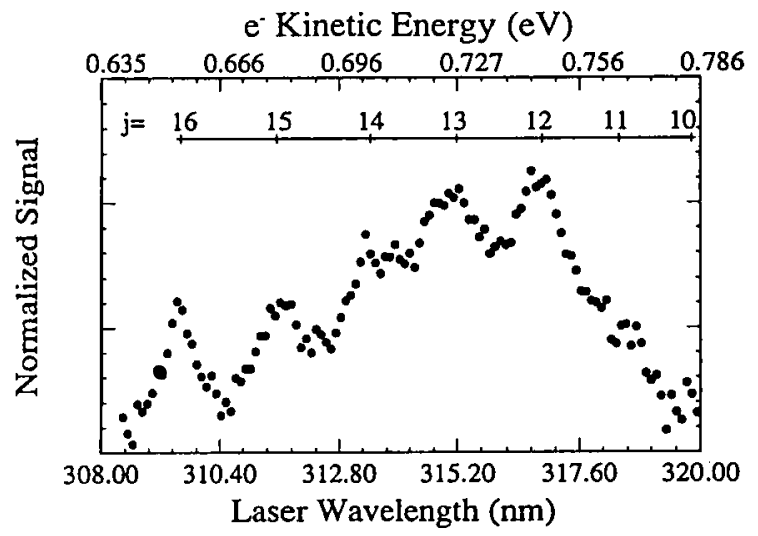

Figure 18. Threshold photodetachment spectrum of $v_{3}^{\prime}=0$ peak in $\mathrm{IHI}^{-}$photoelectron spectrum. Point spacing is $0.1 \mathrm{~nm}$. The energetic thresholds for the asymptotic levels I + HI $(v=0, j=11-16)$ are shown (Ref. 72). 
reasonable that the higher-lying symmetric stretch level of the complex (peak B) should have a shorter lifetime. Similar trends have been observed in collinear scattering calculations on the $\mathrm{Cl}+\mathrm{HCl}^{73}$ and $\mathrm{F}+\mathrm{DBr}^{74}$ reactions.

The observation that the peaks associated with $v_{3}^{\prime}=4$ in Fig. 17 are narrower than the $v_{3}^{\prime}=2$ peaks in Fig. 16 is of interest. The $v_{3}^{\prime}=4$ peaks lie $0.49 \mathrm{eV}$ above the I $+\mathrm{HI}(v=0)$ asymptote compared to $0.30 \mathrm{eV}$ for the levels with $v_{3}^{\prime}=2$, and one might therefore expect the levels with higher total internal energy (the $v_{3}^{\prime}=4$ levels) to dissociate more rapidly. Our result shows that the $v_{3}$ mode really is poorly coupled to dissociation of the complex, as discussed in Section IV.A.3. The narrower peaks for $v_{3}^{\prime}=4$ may be due to the deeper wells in the effective potentials for the higher $v_{3}$ value (see Fig. 8), or because dissociation of the complex through bending motion is less facile for $v_{3}^{\prime}=4$ than for $v_{3}^{\prime}=2$. The possible coupling between the antisymmetric stretch and bending vibration of the complex is discussed further below.

Figure 18 shows the threshold photodetachment spectrum for the $v_{3}^{\prime}=$ 0 levels of the complex. This feature, which appears as a single broad peak in the photoelectron spectrum, actually consists of a series of peaks spaced by an interval that increases from 20 to $25 \mathrm{meV}$ towards lower laser wavelength. This is quite distinct from the $12-\mathrm{meV}$ interval seen in the other spectra. A possible assignment of these peaks is suggested by the approximate correspondence between the peak positions and the energetic thresholds for formation of the asymptotic levels $\mathrm{I}+\mathrm{HI}(v=0$, $j=11-16$ ) from photodetachment of $\mathrm{IHI}^{-}$. These thresholds, shown in Fig. 18, are drawn assuming the threshold for $\mathrm{I}+\mathrm{HI}(v=0, j=0)$ is at $3.79 \mathrm{eV}$, well within the error bars of the value of $E_{b}^{(-)}$for $\mathrm{IHI}^{-}$, $3.80 \pm 0.13 \mathrm{eV}$, used above.

Our results therefore indicate that photodetachment is enhanced near the thresholds for formation of $\mathrm{HI}(v=0)$ in high rotational states. This suggests that the peaks in the spectrum correspond to transitions to hindered rotor levels of the IHI complex which look like nearly free HI rotational states. Because the peak widths are comparable to their spacing, the spectrum implies that internal rotation of the $\mathrm{HI}$ is occurring on the same time scale as dissociation of the IHI $\left(v_{3}=0\right)$ complex. In other words, subsequent to photodetachment, the $\mathrm{H}$ atom, which initially lies between the two I atoms, rotates and becomes associated with one of the I atoms as the complex falls apart. Tannor and co-workers ${ }^{75}$ are currently working on three-dimensional time-dependent wavepacket studies of $\mathrm{IHI}^{-}$ photodetachment which should provide further insight into the dissociation dynamics of IHI $\left(v_{3}=0\right)$.

In his three-dimensional simulation of the $\mathrm{IHI}^{-}$photoelectron spec- 
trum, Schat ${ }^{36}$ observes peaks in the transitions to $v_{3}^{\prime}=0$ levels of the complex which correspond approximately to asymptotic I + HI rotational energy levels. However, his results show significant intensity down to the $\mathrm{I}+\mathrm{HI}(j=0)$ level, suggesting that much lower-lying hindered rotor states of the complex are accessed in the simulation than in the experimental spectrum. Schatz's calculations also show that these hindered rotor levels of IHI dissociate in a rotationally adiabatic manner. ${ }^{34}$ That is, the IHI level formed near the energy asymptote for $\mathbf{I}+\mathrm{HI}(v=0, j)$ predominantly dissociates to $\mathrm{I}+\mathrm{HI}(v=0, j)$. This is physically reasonable if the $\mathrm{HI}$ is already undergoing nearly free rotation in the complex. Hence, our experiment suggests that a more highly rotationally excited HI $(v=0)$ distribution results from dissociation of the complex formed by photodetachment on the real I + HI potential energy surface than on the LEPSA surface used in the simulations. This is likely to be intimately connected with differences in the bending potential and, possibly, the barrier height on the real and model surfaces.

In summary, the $\mathrm{IHI}^{-}$threshold photodetachment results show that, at higher resolution, the $v_{3}^{\prime}=2$ and 4 peaks seen in the photoelectron spectrum are qualitatively different from the $v_{3}^{\prime}=0$ peak. The threshold photodetachment spectrum of the $v_{3}^{\prime}=2$ and 4 features reveals vibrational progressions in quasi-bound symmetric stretch levels of the IHI complex, while the spectrum of the $v_{3}^{\prime}=0$ peak shows what appears to be a progression in internal rotor levels of the IHI complex. The lack of resonance structure in the $v_{3}^{\prime}=0$ peak is reasonable in light of the expectation discussed above that resonances become more pronounced for higher $v_{3}$ levels of the IHI complex.

The absence of the rotational structure underlying the $v_{3}^{\prime}=0$ peak in the $v_{3}^{\prime}=2$ and 4 peaks is more intriguing. It suggests that the bending motion of the complex resembles internal rotation only for the $v_{3}^{\prime}=0$ level. If the effective bend potential is stiffer for the $v_{3}^{\prime}=2$ and 4 levels, and more closely resembles the bending normal vibration in a symmetric triatomic molecule, one might expect photodetachment from $\mathrm{IHI}^{-}\left(v_{2}^{\prime \prime}=\right.$ 0 ) to have good Franck-Condon overlap only with the lowest bending level associated with these antisymmetric stretch levels of the IHI complex. This implies the effective bending potential depends strongly on the $v_{3}$ quantum number. One certainly might expect coupling between the bending and antisymmetric stretch vibrations in the complex, since both are essentially $\mathrm{H}$ atom vibrations. This has been explored in a recent paper by Kubach, ${ }^{76}$ in which the eigenvalues and eigenfunctions of the twodimensional antisymmetric stretch/bend Hamiltonian are determined as a function of interiodine distance.

We now consider the implications of the higher resolution $\mathrm{IHI}^{-}$results 
for the analysis of the $\mathrm{BrHBr}^{-}$photoelectron spectrum. A comparison of the $\mathrm{IHI}^{-}$simulations in Fig. 15 shows that the adiabatic bend approximation used in the $\mathrm{BrHBr}^{-}$analysis does a reasonably accurate job reproducing the positions of resonances. However, this approximation is clearly inadequate if the complex dissociates in a noncollinear manner, and it therefore fails to reproduce the overall width and underlying rotational structure of the $v_{3}^{\prime}=0$ peak in the exact three-dimensional simulation.

As discussed in Section IV.B, the best-fit $\mathrm{Br}+\mathrm{HBr}$ surface was designed to reproduce the broad $v_{3}^{\prime}=0$ and 2 experimental peaks assuming these were single, homogeneous peaks. If these peaks consist of unresolved progressions of the type predicted (and experimentally observed) for the $v_{3}^{\prime}=0$ peak in the $\mathrm{IHI}^{-}$spectrum, then some revision of the proposed $\mathrm{Br}+\mathrm{HBr}$ surface will be required. In particular, it should be possible to fit the data with a surface which is less repulsive in the FranckCondon region. It is clearly worthwhile to obtain the threshold photodetachment spectrum of $\mathrm{BrHBr}^{-}$to see if any of the broad peaks exhibit underlying structure at higher resolution, and these experiments will begin soon in our laboratory.

\section{ASYMMETRIC HYDROGEN EXCHANGE REACTIONS}

\section{A. General Considerations}

This section describes the application of photodetachment spectroscopy to studies of the transition state region for reactions $A+\mathrm{HB} \rightarrow \mathrm{HA}+\mathrm{B}$, where $A \neq B$. A and $B$ can be atomic or polyatomic species. These asymmetric reactions are of considerable chemical interest. In particular, studies of reactions in which A and B are (unlike) halogen atoms have led to the formulation of many fundamental concepts in reaction dynamics. Several of the first product state-resolved experimental studies in chemical dynamics were performed on these reactions. ${ }^{77,78}$ On the theoretical side, classical $^{79-83}$ and quantum mechanical ${ }^{74,84,85}$ scattering calculations have been carried out on model potential energy surfaces in order to understand the experimental results. Studies by Manz and co-workers ${ }^{53,74,86}$ on the $\mathrm{F}+\mathrm{DBr}$ reaction predict sharp resonance structure, just as in the symmetric reactions.

Asymmetric hydrogen exchange reactions are therefore a tempting target for photodetachment studies, since asymmetric $\mathrm{AHB}^{-}$anions are often as strongly hydrogen-bonded as the symmetric $\mathrm{BrHBr}^{-}$and $\mathrm{IHI}^{-}$anions. However, for an asymmetric reaction, one must consider the geometric overlap between the anion and neutral transition state more carefully than 
for a symmetric reaction. This point has been emphasized by Brauman and co-workers ${ }^{6}$ in their study of $\mathrm{ROHF}^{-}$anions.

For the $\mathrm{Br}+\mathrm{HBr} / \mathrm{BrHBr}^{-}$system, the $\mathrm{BrHBr}^{-}$anion is linear and centrosymmetric, and the major concern from the point of view of geometric overlap is how close the equilibrium interhalogen distance in the anion is to the saddle point geometry on the neutral surface. For an asymmetric reaction such as $\mathrm{Br}+\mathrm{HI} \rightarrow \mathrm{HBr}+\mathrm{I}$, one must also consider the location of the $\mathrm{H}$ atom in $\mathrm{BrHI}^{-}$, as this will determine whether photodetachment primarily accesses the reactant or product valley on the neutral potential energy surface. To first order, the $\mathrm{H}$ atom location is determined by the relative proton affinities of $\mathrm{Br}^{-}$and $\mathrm{I}^{-}$. Since the proton affinity of $\mathrm{Br}^{-}$ is $0.40 \mathrm{eV}$ higher than that of $\mathrm{I}^{-},{ }^{87}$ one expects the ion to look like $\mathrm{I}^{-} \cdot \mathrm{HBr}$, and a vertical photodetachment transition from the equilibrium geometry of the anion should land in the $\mathrm{I}+\mathrm{HBr}$ product valley of the reaction. The photoelectron spectra of $\mathrm{BrHI}^{-}, \mathrm{FHI}^{-}$, and $\mathrm{FHBr}^{-}$will be discussed in Sections V.B and V.C as examples of asymmetric bihalide systems.

We have also studied the reaction $\mathrm{F}+\mathrm{CH}_{3} \mathrm{OH} \rightarrow \mathrm{HF}+\mathrm{CH}_{3} \mathrm{O}$ by photoelectron spectroscopy of $\mathrm{CH}_{3} \mathrm{OHF}^{-}$. In this case, the proton affinity of $\mathrm{CH}_{3} \mathrm{O}^{-}$is $0.42 \mathrm{eV}$ higher than that of $\mathrm{F}^{-},{ }^{87}$ the anion should look like $\mathrm{CH}_{3} \mathrm{OH} \cdot \mathrm{F}^{-}$, and photodetachment should access the reactant valley and saddle-point region of the neutral potential energy surface. The photoelectron spectrum of $\mathrm{CH}_{3} \mathrm{OHF}^{-}$is presented in Section V.D.

\section{B. Photoelectron Spectroscopy of $\mathrm{BrHI}^{-}$}

\section{Results and Qualitative Analysis}

Figure 19 shows the photoelectron spectra of $\mathrm{BrHI}^{-}$and $\mathrm{BrDI}^{-}$taken at $213 \mathrm{~nm} .{ }^{55}$ Each spectrum shows two progressions of approximately evenly spaced peaks. The peaks labelled $\mathrm{A}$ and $\mathrm{A}^{*}$ occur at the same electron kinetic energy in both spectra and are taken to be band origins of the progressions. The peak spacing within each progression in Fig. 19 is noticeably less in the $\mathrm{BrDI}^{-}$spectrum than in the $\mathrm{BrHI}^{-}$spectrum. The direction of this isotope shift shows we are observing progressions in the neutral $\mathrm{BrHI}$ complex in a vibrational mode primarily involving $\mathrm{H}$ atom motion. This is assigned to the $v_{3}$ stretching mode of the BrHI complex. The $\mathrm{A}-\mathrm{A}^{*}$ separation in each spectrum is $0.90 \pm 0.02 \mathrm{eV}$ $\left(7300 \pm 200 \mathrm{~cm}^{-1}\right)$. This is slightly less than the spin-orbit splitting in atomic I $\left(7600 \mathrm{~cm}^{-1}\right)$ and suggests that the two progressions with band origins $\mathrm{A}$ and $\mathrm{A}^{*}$ correspond to two electronic states of the $\mathrm{BrHI}$ complex which asymptotically correlate to $\mathrm{HBr}+\mathrm{I}\left({ }^{2} \mathrm{P}_{3 / 2}\right)$ and $\mathrm{HBr}+\mathrm{I}^{*}\left({ }^{2} \mathrm{P}_{1 / 2}\right)$, respectively. The arrows at 2.07 and $1.36 \mathrm{eV}$ show the electron kinetic energies corresponding to formation of $\mathrm{I}+\mathrm{HBr}(v=0)$ ground-state prod- 

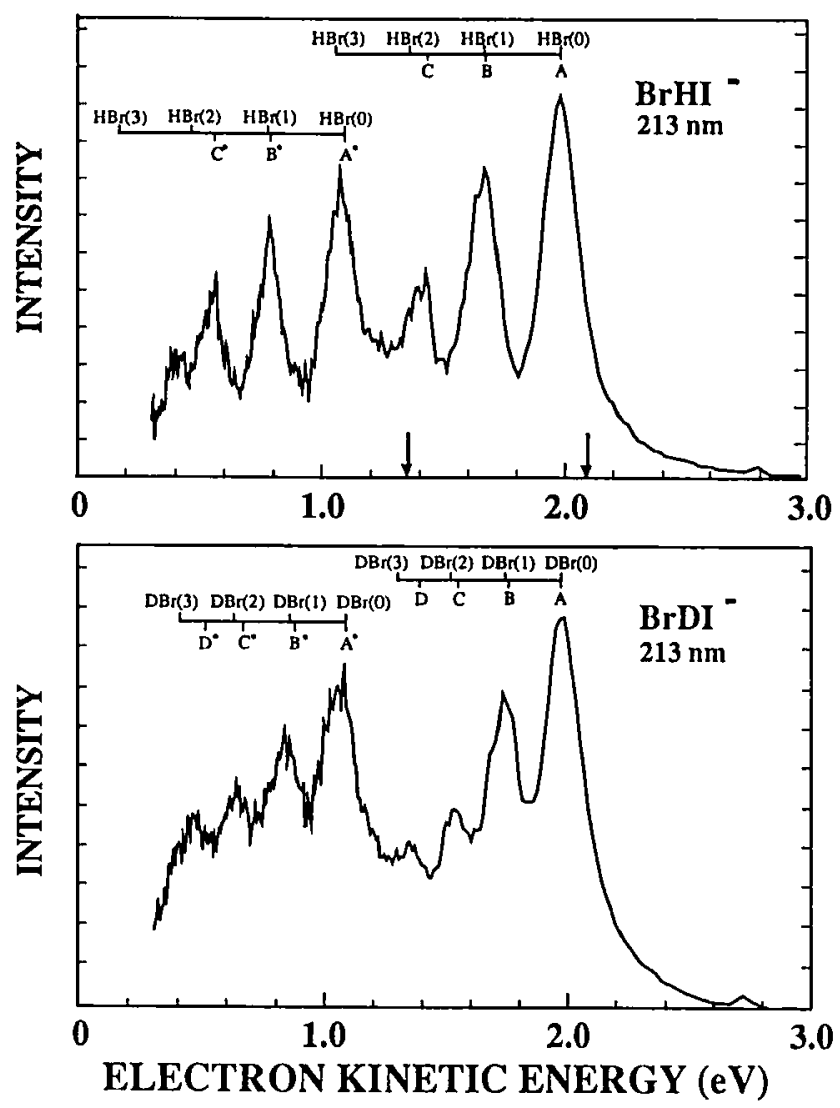

Figure 19. Photoelectron spectra of $\mathrm{BrHI}^{-}$and $\mathrm{BrDI}^{-}$at $213 \mathrm{~nm}$. Arrows at 2.07 and $1.36 \mathrm{eV}$ show thresholds for formation of ground-state products $\mathrm{I}\left({ }^{2} \mathrm{P}_{3 / 2}\right)+\mathrm{HBr}(v=0)$ and ground-state reactants $\mathrm{Br}\left({ }^{2} \mathrm{P}_{3 / 2}\right)+\mathrm{HI}(v=0)$, respectively. These thresholds are referred to in the text as product and reactant asymptotes. The spacing between the first few $\mathrm{HBr}$ and $\mathrm{DBr}$ vibrational levels is shown at the top of each spectrum.

ucts and $\mathrm{Br}+\mathrm{HI}(v=0)$ ground-state reactants, respectively. All the peaks are due to states of the neutral complex with sufficient energy to dissociate to ground-state products.

A comparison of the $\mathrm{BrHI}^{-}$and $\mathrm{BrDI}^{-}$spectrum with the symmetric bihalide spectra discussed previously shows two important differences. In the symmetric bihalide spectra, large variations in the peak widths were observed. In contrast, the peaks in Fig. 19 are all quite broad. In the $\mathrm{BrHI}^{-}$spectrum, for example, the peak widths in the ground state progression are $\sim 170 \mathrm{meV}$, somewhat wider than the peaks in the excited state progression $(\sim 140 \mathrm{meV})$. Secondly, the symmetric $\mathrm{XHX}^{-}$spectra showed 


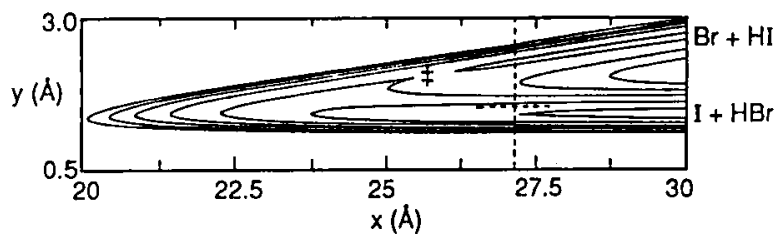

Figure 20. Effective collinear LEPS potential energy surface for $\mathrm{Br}+\mathrm{HI}$ reaction obtained from three-dimensional surface of Ref. 83. The surface is plotted using mass-weighted coordinates defined in Eq. (20). The skew angle (see Eq. 11) is $8.2^{\circ}$. The saddle point is marked by $\ddagger$. The estimated $\mathrm{BrHI}^{-}$equilibrium geometry (see text) is at the intersection of the dashed lines (Ref. 55).

a substantial "red shift" in the $v_{3}$ frequency relative to diatomic $\mathrm{HX}$. Figure 19 shows that the peak spacings in the $\mathrm{BrHI}^{-}$and $\mathrm{BrDI}^{-}$spectra closely parallel the first few vibrational energy levels $\mathrm{HBr}$ and $\mathrm{DBr}$. The peak spacings are, on the average, only $200 \mathrm{~cm}^{-1}$ less than the vibrational frequency of $\mathrm{HBr}$ or $\mathrm{DBr}$.

The differences between the $\mathrm{BrHI}^{-}$and $\mathrm{BrDI}^{-}$spectra and the symmetric bihalide spectra will now be explored within the framework of the time-independent and time-dependent methods discussed in the previous sections, with the ultimate goal of simulating the ground-state progressions in the spectra. The $\mathrm{BrHI}^{-}$and $\mathrm{BrDI}^{-}$anions are not well characterized; only the $v_{3}$ fundamentals have been measured in a matrix ${ }^{88}$ (920 and $728 \mathrm{~cm}^{-1}$, respectively). No high-level $a b$ initio calculations have been performed on $\mathrm{BrHI}^{-}$to determine the ion geometry, although the ions are expected to be linear, based on ab initio studies of $\mathrm{FHCl}^{-}$and $\mathrm{FHBr}^{-} .89 \mathrm{~A}$ LEPS potential energy surface for the $\mathrm{Br}+\mathrm{HI}$ reaction has been proposed by Broida and Persky ${ }^{83}$ (the BP surface). This surface was constructed on the basis of quasi-classical trajectory calculations which reproduce the reaction rate constant ${ }^{90}$ and $\mathrm{HBr}$ product vibrational distribution. ${ }^{77} \mathrm{We}$ use the BP surface in our simulations without modification and vary the anion geometry (see below) to achieve optimal agreement with the experimental photoelectron spectra. This yields a qualitative understanding of the features in the photoelectron spectra. In order to actually extract new information on the $\mathrm{Br}+\mathrm{HI}$ surface from our spectra, further characterization of the ion geometry is required.

The BP surface has a collinear minimum energy path and a $0.88 \mathrm{~kJ} / \mathrm{mol}$ barrier which lies in the $\mathrm{Br}+\mathrm{HI}$ reactant channel. Figure 20 shows the effective collinear potential energy surface obtained from the three-dimensional BP surface. The surface is plotted using the mass-weighted coordinates $x$ and $y$, where 


$$
\begin{aligned}
x=\sqrt{\frac{\mu_{\mathrm{I}, \mathrm{HBr}}}{\mu_{\mathrm{HBr}}}} R_{\mathrm{I}, \mathrm{HBr}} & \approx 7.0 R_{\mathrm{I}-\mathrm{Br}} \\
y & =R_{\mathrm{H}-\mathrm{Br}}
\end{aligned}
$$

Here $R_{\mathrm{I}, \mathrm{HBr}}$ is the separation between the $\mathrm{I}$ atom and the $\mathrm{HBr}$ center of mass. Note that for the heavy + light-heavy mass combination, $x^{2} \gg y^{2}$, and therefore $x \approx \rho$ (see Eq. 13).

The mass-weighted coordinates $x$ and $y$ are assumed to be proportional to the normal coordinates for the $v_{1}$ and $v_{3}$ modes, respectively, for the anion. The $v_{3}$ stretch potential for the anion is approximated by a Morse potential:

$$
U(y)=D_{e}\left(1-\exp \left\{-\beta\left[y-R_{e}(\mathrm{HBr})\right]\right\}\right)^{2}
$$

Here $R_{e}(\mathrm{HBr})$ is the equilibrium $\mathrm{H}-\mathrm{Br}$ separation in the anion. $D_{e}$ and $\beta$ are fixed by the matrix isolation values for the $v_{3}$ frequency in $\mathrm{BrHI}^{-}$ and $\mathrm{BrDI}^{-}{ }^{88}$ The $v_{1}$ potential is assumed to be a harmonic oscillator potential with frequency $100 \mathrm{~cm}^{-1}$ centered at $R_{e}(\mathrm{IBr})$, the equilibrium interhalogen distance in the anion. The values of $R_{e}(\mathrm{IBr})$ and $R_{e}(\mathrm{HBr})$ affect the peak spacings and intensities, respectively, in the simulated photoelectron spectrum, and were adjusted in order to optimize agreement between a one-dimensional simulation and experiment. ${ }^{55}$ The best agreement is obtained for $R_{e}(\mathrm{IBr})=3.88 \AA \quad\left(x_{e}=27.1 \AA\right.$, Eq. 20) and $R_{e}(\mathrm{HBr})=1.55 \AA$. This assumed equilibrium geometry for the anion is indicated in Fig. 20 at the intersection of the dashed lines.

The anion equilibrium geometry shown in Fig. 20 indicates that photodetachment primarily accesses the $\mathrm{I}+\mathrm{HBr}$ product valley, as expected based on the relative proton affinities of $\mathrm{I}^{-}$and $\mathrm{Br}^{-}$. In this region of the potential energy surface, the nascent $\mathrm{HBr}$ bond is nearly fully formed. This is consistent with the experimental observation that the peak spacing in the $v_{3}$ progression is only slightly less than the vibrational frequency in diatomic $\mathrm{HBr}$. Good geometric overlap with the product valley is also consistent with the overall intensity distribution in the spectra; the most intense peak occurs near the $\mathrm{I}+\mathrm{HBr}$ product asymptote (arrow at $2.07 \mathrm{eV}$ in Fig. 19), and the peak intensity decreases as the $\mathrm{Br}+\mathrm{HI}$ reactant asymptote (arrow at $1.36 \mathrm{eV}$ ) is approached.

These points can be illustrated further with the aid of Fig. 21, which shows the $v_{3}$ potential for the anion (Eq. 22) and the approximate $v_{3}$ potential for the neutral resulting from taking a cut through the BP potential energy surface at $x=x_{e}$. The latter is an asymmetric double minimum 


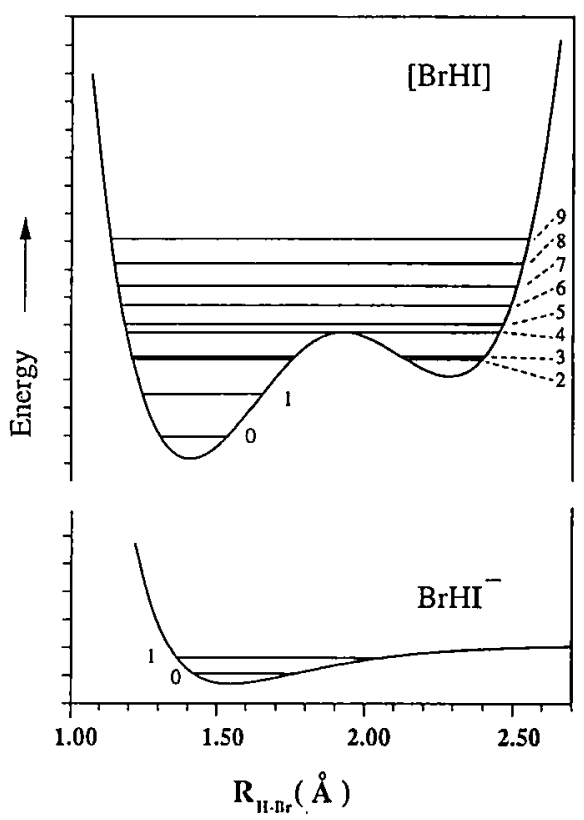

Figure 21. Anion and neutral $v_{3}$ potentials used in one-dimensional analysis of $\mathrm{BrHI}^{-}$spectrum. Eigenstates are labelled by $v_{3}$ quantum number. Tick marks on vertical axis are spaced by $0.2 \mathrm{eV}$ (Ref. 55).

potential with the minimum in the $\mathrm{I}+\mathrm{HBr}$ valley lying $0.58 \mathrm{eV}$ below the minimum in the $\mathrm{Br}+\mathrm{HI}$ valley. The first few eigenvalues of both potentials are shown. A one-dimensional Franck-Condon simulation (see Ref. 55) shows that the $v_{3}^{\prime \prime}=0$ level of the anion has the best Franck-Condon overlap with the neutral $v_{3}^{\prime}=0,1$, and 3 levels; these are localized in the $\mathrm{I}+\mathrm{HBr}$ valley and look like perturbed $\mathrm{HBr}$ vibrational levels. Transitions to the $v_{3}^{\prime}=0,1$, and 3 levels result in the three highest energy peaks in the $\mathrm{BrHI}^{-}$photoelectron spectrum. The one-dimensional analysis predicts the $v_{3}^{\prime}=0$ peak to be the most intense, in agreement with experiment. Although the $v_{3}^{\prime}=2$ and 3 levels are nearly degenerate, the $v_{3}^{\prime}=2$ eigenfunction is localized in the $\mathrm{Br}+\mathrm{HI}$ well and has very little overlap with the $\mathrm{BrHI}^{-}\left(v_{3}^{\prime \prime}=0\right)$ level.

We next consider the origin of the uniformly broad peaks in the $\mathrm{BrHI}^{-}$ and $\mathrm{BrDI}^{-}$spectra. In contrast to the photodetachment of symmetric bihalides, photodetachment of $\mathrm{BrHI}^{-}$accesses a more repulsive region of the potential energy surface, namely, the exit valley of an exothermic reaction. One therefore expects rapid dissociation of the BrHI complex, consistent with the broad peaks observed in the photoelectron spectra.

This point can be explored in more detail with reference to Fig. 22, which shows the vibrationally adiabatic potentials $\epsilon_{v_{3}}(\rho)$ obtained by solving Eq. (16) for the potential energy surface in Fig. $20 .{ }^{91}$ (Recall $\rho \approx x$ for this reaction.) These curves correlate asymptotically to various $\mathbf{I}+$ 
Figure 22. Vibrationally adiabatic curves $\epsilon_{v_{3}}(\rho)$ (Eq. 16) for $\mathrm{Br}+\mathrm{HI}$ reaction derived from surface in Fig. 20. The vertical dashed line indicates $\rho_{e}$ for $\mathrm{BrHI}^{-}$. The level numbering is the same as in Fig. 21. The $\mathrm{Br}+\mathrm{HI}(v=0,1)$ and $\mathrm{I}+\mathrm{HBr}(v=0-3)$ asymptotic levels are indicated. The apparent intersections of some of the curves are actually weakly avoided crossings.

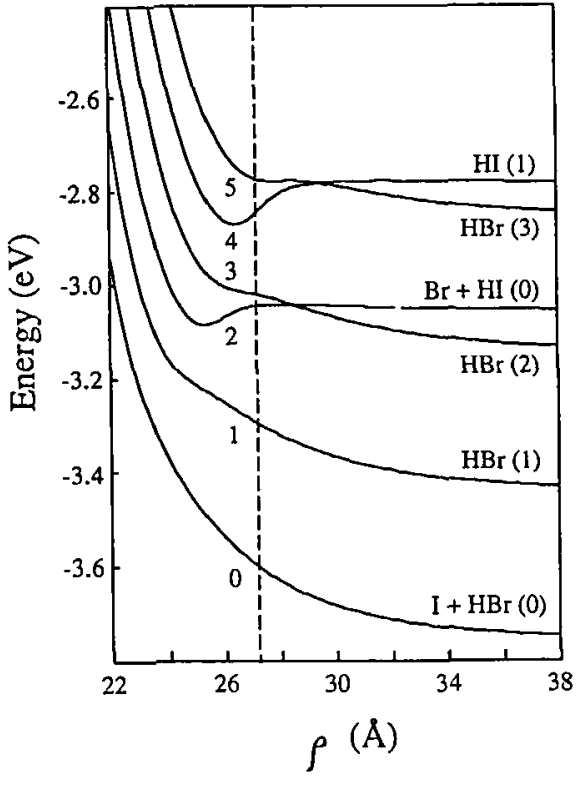

$\mathrm{HBr}(v)$ and $\mathrm{Br}+\mathrm{HI}(v)$ levels, as indicated in Fig. 22. The dashed vertical line in Fig. 22 indicates $\rho_{e}$ for $\mathrm{BrHI}^{-}$. The intersections of the adiabatic curves with this line are the $v_{3}$ energy levels of the BrHI complex shown in Fig. 21, and the curves are numbered accordingly. As discussed above, the $v_{3}^{\prime \prime}=0$ level of $\mathrm{BrHI}^{-}$has good Franck-Condon overlap with the $v_{3}^{\prime}$ $=0,1$, and 3 levels. The corresponding adiabatic curves are repulsive, and transitions to these curves should yield broad peaks in the photoelectron spectrum. Note that these curves correlate diabatically with the $\mathrm{I}+\mathrm{HBr}(v=0,1$, and 2$)$ product states. While several of the adiabatic curves $(2,4)$ have wells which could support resonance states, the $v_{3}$ wavefunctions near $\rho_{e}$ for these curves are localized in the $\mathrm{Br}+\mathrm{HI}$ valley and have little overlap with the ground state of $\mathrm{BrHI}^{-}$. The curves with wells in the transition state region all correlate diabatically to $\mathrm{Br}+\mathrm{HI}$ reactant states.

\section{Time-Dependent Simulations of the Spectra}

In this section, the two-dimensional time-dependent wavepacket analysis discussed in Sections III.B and IV.A.5 will be used to simulate the ground electronic state progressions in the $\mathrm{BrHI}^{-}$and $\mathrm{BrDI}^{-}$photoelectron spectra and to probe the underlying dynamics. ${ }^{55}$ Figure 23 shows the initial wavepacket, $\phi(t=0)$, which results from projecting the ground-state vibrational wavefunction of $\mathrm{BrHI}^{-}$onto the $\mathrm{BP}$ effective collinear potential 

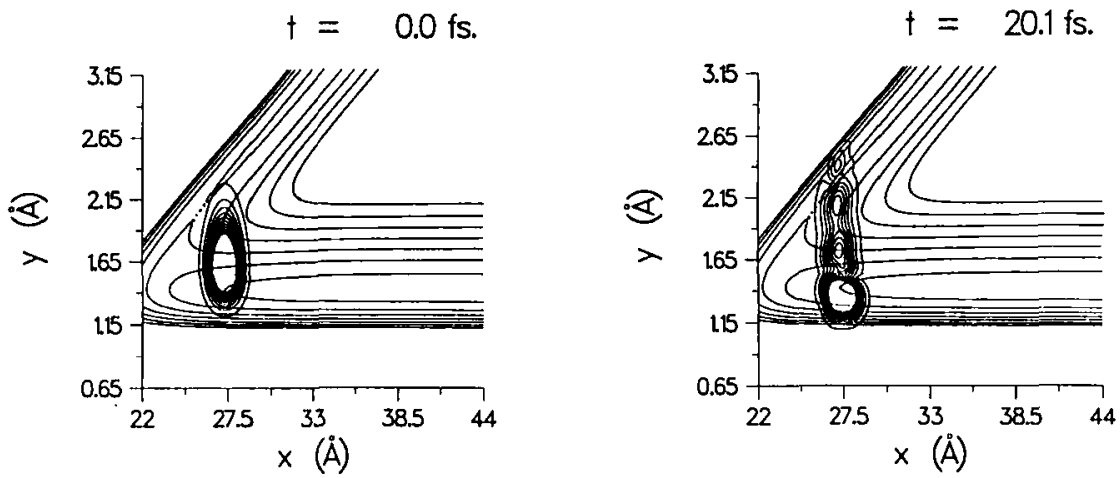

(a)
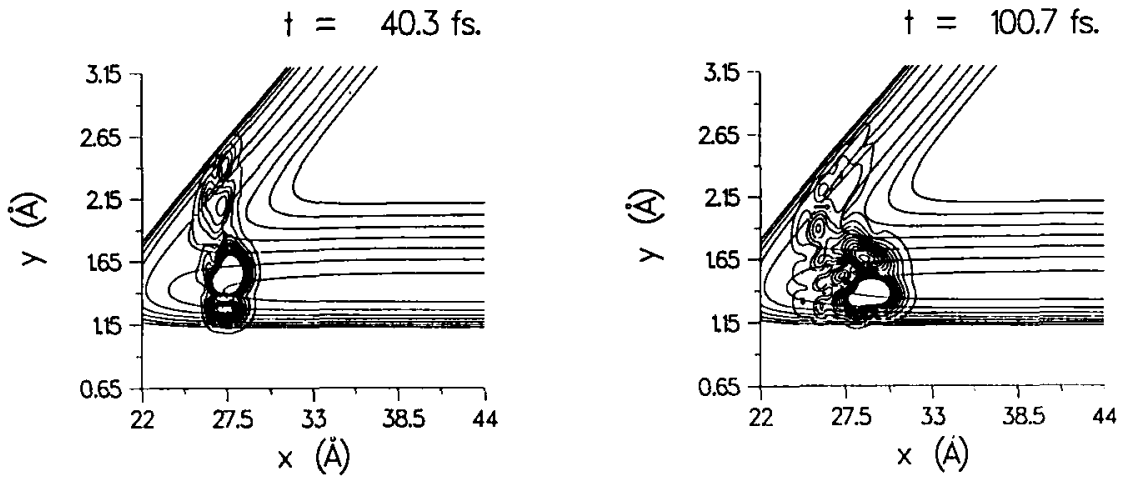

(b)

Figure 23. Evolution of initially prepared wavepacket $\phi(0)$ on $\mathrm{Br}+\mathrm{HI}$ potential energy surface shown in Fig. 20 (Ref. 55).

energy surface. This shows that photodetachment primarily accesses the product side of the $\mathrm{Br}+\mathrm{HI}$ surface, as discussed in the previous sections. However, $\phi(0)$ does have some amplitude at the saddle-point region of the potential energy surface and therefore will have small but nonzero overlap with states localized in the $\mathrm{Br}+\mathrm{HI}$ valley.

Figure 23 also shows the time evolution of the initial wavepacket on the $\mathrm{Br}+\mathrm{HI}$ surface. At early times $(t<60 \mathrm{fs})$, we observe rapid oscillation of the wavepacket along the $y\left(v_{3}\right)$ coordinate, and slower movement of the wavepacket along the $x$ coordinate. While the wavepacket in this time interval has some amplitude in the $\mathrm{Br}+\mathrm{HI}$ reactant valley, the bulk of the wavepacket remains in the $\mathrm{I}+\mathrm{HBr}$ product valley. This is in contrast to the wavepacket simulations on our best-fit $\mathrm{Br}+\mathrm{HBr}$ surface (Fig. 10). In those simulations, due to the higher symmetry of the anion and neutral, 

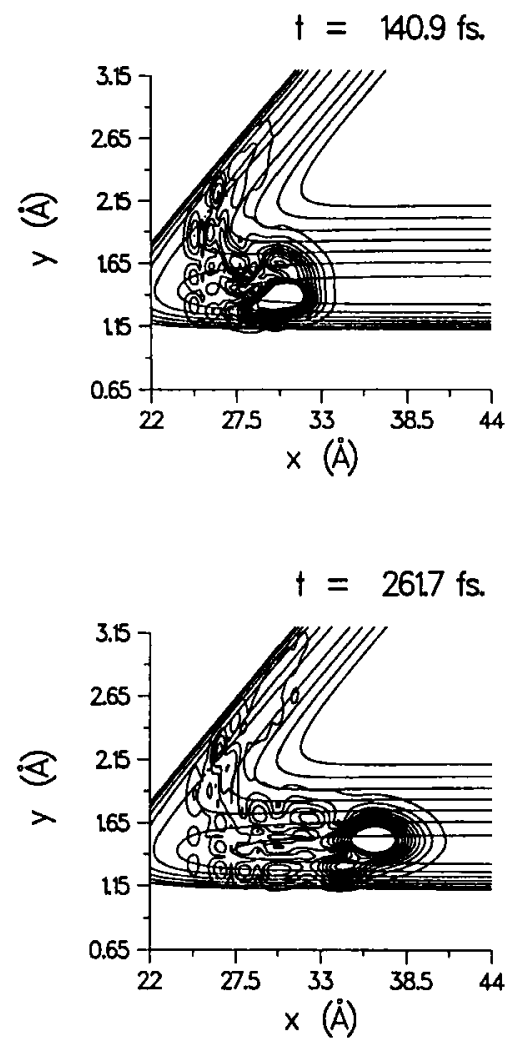

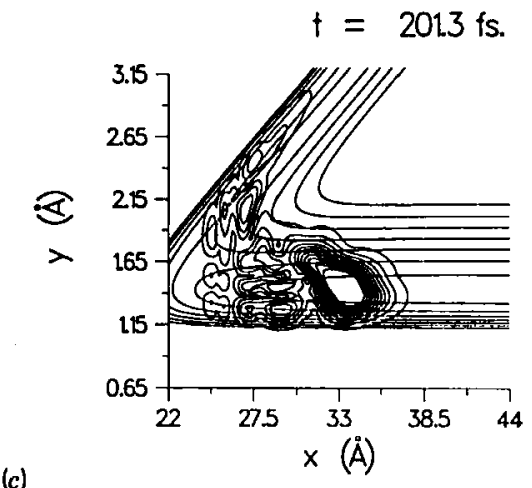

(c)

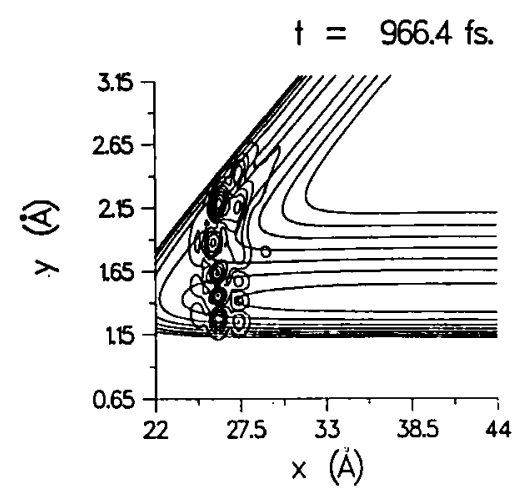

(d)

Figure 23. (Continued).

the wavepacket splits evenly between the two valleys of the $\mathrm{Br}+\mathrm{HBr}$ potential energy surface.

At longer times, $t>60 \mathrm{fs}$, several trends are noticeable. By this time, most of the wavepacket has moved out of the initial region accessed by photodetachment, so we expect $\langle\phi(t) \mid \phi(0)\rangle$ to be small. Most of the wavepacket remains confined to the $\mathrm{I}+\mathrm{HBr}$ product valley. The spreading of this portion of the wavepacket along the $x$ coordinate is quite evident for $t>200 \mathrm{fs}$; from the leading edge (at higher $x$ ) to the tail there is an increasing number of nodes along the $y$ coordinate. This suggests that those states localized in the product valley with higher $v_{3}$ excitation dissociate more slowly. This effect is consistent with the observation that the repulsive adiabatic curves in Fig. 22 are less steep at higher levels of excitation. 

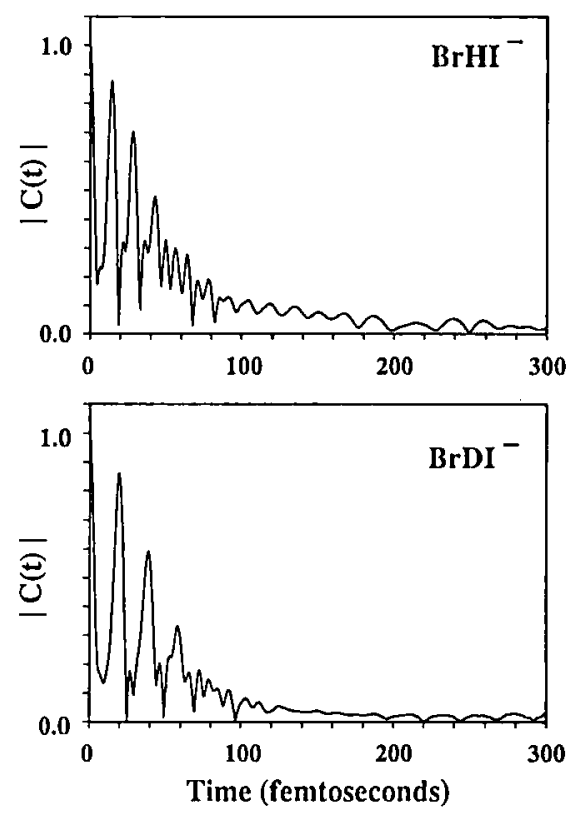

Figure 24. Modulus of time-autocorrelation function $|C(t)|$ obtained from wavepacket propagation on $\mathrm{Br}+\mathrm{HI}$ surface shown in Fig. 20 (Ref. 55).

Even at the longest time, $t=966 \mathrm{fs}$ (Fig. 23d), the wavepacket still has some amplitude near the transition state region. This occurs because the BP potential energy surface does support resonances, and some of the quasi-bound states responsible for these resonances have nonzero overlap with the ground state of the anion. The wavefunction in Fig. $23 d$ represents a superposition of these quasi-bound states, but the most noticeable feature of this wavefunction is the four nodes along the $y$ coordinate. This indicates that $v_{3}=4$ for the dominant resonance state.

Figure 24 shows the modulus of the autocorrelation function $|C(t)|(\mathrm{Eq}$. 8 ) obtained from propagating $\phi(0)$ on the BP surface. The correspondence between the wavefunctions in Fig. 23 and $|C(t)|$ is quite clear. At times $t<60 \mathrm{fs},|C(t)|$ exhibits a fast oscillation whose period corresponds to the $v_{3}$ motion of the BrHI complex. This decays after about $60 \mathrm{fs}$, indicating that dissociation of the complex is occurring on this time scale. Thus, as in the $\mathrm{Br}+\mathrm{HBr}$ analysis, the simulations show fast vibrational motion, primarily involving the $\mathrm{H}$ atom, occurring as the complex dissociates. At longer times $(t>200 \mathrm{fs})$, we see that $|C(t)|$ does not become zero, but instead shows a persistent oscillation. This results from the small resonance contribution to the simulated spectrum; Fig. 24 shows that even at $t=$ $300 \mathrm{fs}$, there is some residual overlap with the initial wavepacket due to quasi-bound states localized near the transition state region.

Finally, we simulate the $\mathrm{BrHI}^{-}$and $\mathrm{BrDI}^{-}$photoelectron spectra by 
Figure 25. Simulated photoelectron spectra (solid) for (top) $\mathrm{BrHI}^{-}$and (bottom) $\mathrm{BrDI}^{-}$from two-dimensional calculation. The simulations are shifted so that the $0-0$ transitions line up with the experimental spectra (-) and have been convoluted with the instrumental resolution function (Ref. 55).
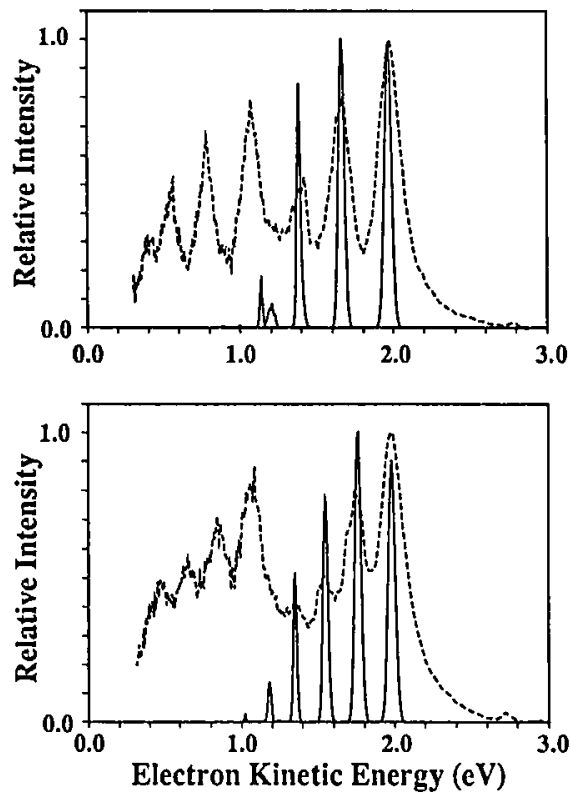

taking the Fourier transform of $C(t)$ (Eq. 7). The results convoluted with our experimental resolution are shown in Fig. 25. The five peaks in the $\mathrm{BrHI}^{-}$simulation result from transitions to the $v_{3}^{\prime}=0,1,3,4$, and 5 levels of the complex. The intensity of the transition to the $v_{3}^{\prime}=2$ level is too small to be seen. Figure 22 shows that the vibrationally adiabatic curve for the $v_{3}^{\prime}=4$ level, which diabatically correlates to $\mathrm{Br}+\mathrm{HI}(v=1)$, has a well which should support resonances. Indeed, if we Fourier transform $C(t)$ assuming a somewhat higher instrumental resolution of $4 \mathrm{meV},{ }^{55}$ we find the weak $v_{3}^{\prime}=4$ peak at $1.2 \mathrm{eV}$ does consist of a progression of closely spaced $v_{1}$ levels of the complex. However, the overall contribution of resonances to the simulation is quite small, as expected based on our wavepacket dynamics calculations.

The first three peaks in the $\mathrm{BrHI}^{-}$simulation (those at highest electron kinetic energy) line up well with the experimental peaks, as do the first four peaks in the $\mathrm{BrDI}^{-}$simulation. The excited state progression in the experimental spectra makes it difficult to compare the remaining simulated peaks with experiment, although shoulders appear on the high-energy side of the first excited-state peak in each spectrum near the energies of the lowest energy peaks in the simulations. As discussed above, the agreement between the peak positions and intensities in the experimental and simulated spectra results from assuming the BP surface is correct and optimizing the anion geometry. The analysis therefore shows that we can reproduce 
experimental peak positions and intensities using a reasonable ion geometry and neutral potential energy surface.

However, the peak widths in the simulations are considerably narrower than the experimental peak widths. Here, as in the $\mathrm{BrHBr}^{-}$analysis, one must consider if this discrepancy results from deficiencies in the BP surface, or if the effective collinear approximation artificially constrains the peak widths as it only allows for collinear decay of the complex. This can clearly be answered by performing three-dimensional simulations of the $\mathrm{BrHI}^{-}$spectrum using the same anion geometry and neutral potential energy surface.

\section{Excited Electronic States in the BrHI- Photoelectron Spectrum}

As discused in Section V.B.1, the photoelectron spectra of $\mathrm{BrHI}^{-}$and $\mathrm{BrDI}^{-}$show an additional progression which has been assigned to an excited state of the complex that asymptotically correlates to spin-orbit excited $I^{*}\left({ }^{2} \mathrm{P}_{1 / 2}\right)+\mathrm{HBr}(\mathrm{DBr})$. This section discusses the role of excited electronic states in these photoelectron spectra in more detail. The effects of spin-orbit excitation in the $\mathrm{Br}+\mathrm{HI}$ and $\mathrm{F}+\mathrm{HBr}$ reactions have been studied previously by Bergmann ${ }^{92}$ and Hepburn, ${ }^{93}$ respectively.

Figure 26 shows an electronic correlation diagram for the $\mathrm{Br}+\mathrm{HI}$ reaction, assuming $C_{x_{v}}$ symmetry. Let us consider the product side of this diagram, since this is the region of the $\mathrm{Br}+\mathrm{HI}$ surface most relevant to our experiment. In the asymptotic region of the product valley, the ground- and first-excited electronic state are separated by the spin-orbit splitting between the $I\left({ }^{2} P_{3 / 2}\right)$ and $I^{*}\left({ }^{2} P_{1 / 2}\right)$ states, $7600 \mathrm{~cm}^{-1}$. Closer to the transition state region, the $\mathrm{HBr}$ perturbs the spherical symmetry of the I atom. In this region, Hund's case (c) coupling is appropriate: $J$, the electronic and spin-angular momentum of the I atom, is no longer a good quantum number, but $\Omega$, the projection of $J$ on the internuclear axis, is a good quantum number. The interaction of $\mathrm{HBr}$ with a ground state I atom splits the degenerate $\mathrm{I}\left({ }^{2} \mathrm{P}_{3 / 2}\right)$ state into the $\mathrm{X}(\Omega=1 / 2)$ and $\mathrm{I}(\Omega=$ $3 / 2)$ states, and the $\mathrm{II}(\Omega=1 / 2)$ state results from the interaction of $\mathrm{HBr}$ with $I^{*}\left({ }^{2} P_{1 / 2}\right)$. At even smaller interhalogen distances, where all three atoms strongly interact, Hund's case (a) coupling is more appropriate. The $X(1 / 2)$ and $I I(1 / 2)$ states are mixed, yielding the ${ }^{2} \Sigma_{2}$ and ${ }^{2} \Pi_{1 / 2}$ states in the diagram, while the $\mathrm{I}(3 / 2)$ state is more appropriately labelled as ${ }^{2} \Pi_{3 / 2}$. The two ${ }^{2} \Pi$ states correlate diabatically to excited-state reactants and should lie much higher than the ${ }^{2} \Sigma_{1 / 2}$ state in this region.

Since the energy interval between the two progressions in the $\mathrm{BrHI}^{-}$ and $\mathrm{BrDI}^{-}$photoelectron spectra, $7300 \mathrm{~cm}^{-1}$, is very close to the asymptotic spin-orbit splitting in atomic I, it appears that Hund's case (c) coupling is appropriate for describing the electronic states probed in our experi- 


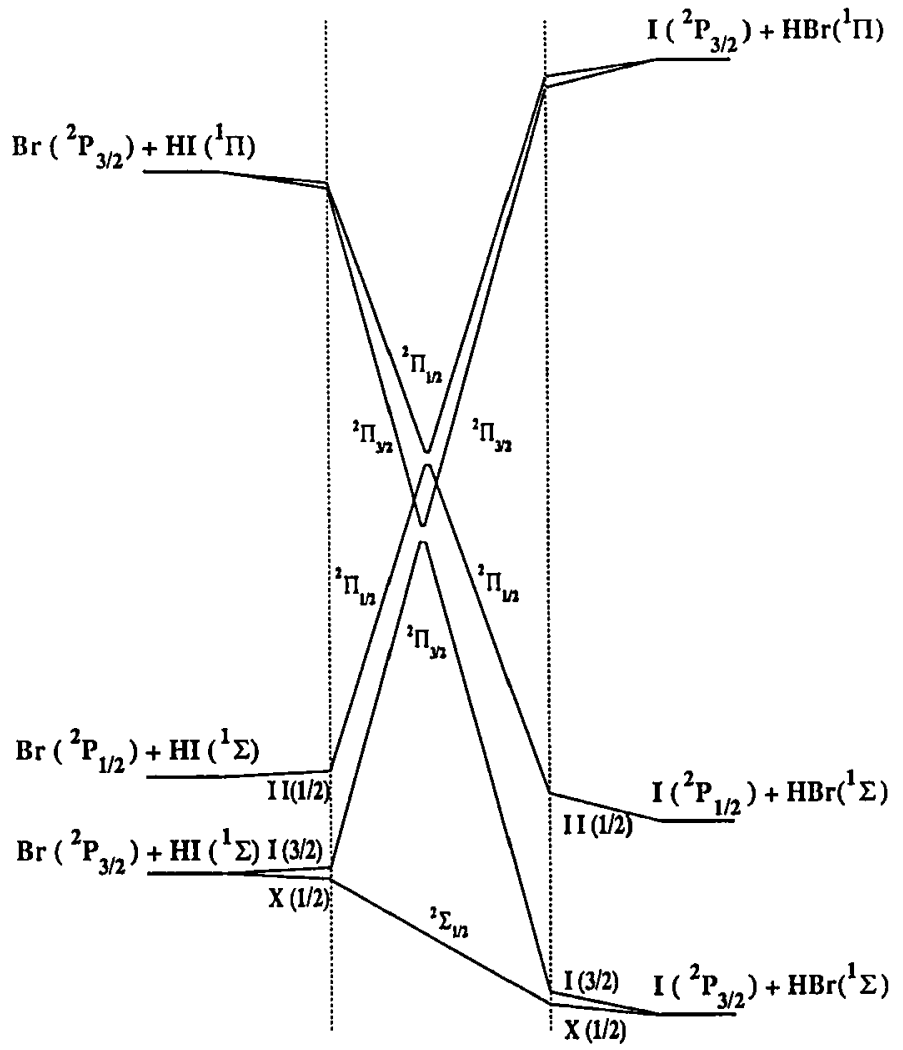

Figure 26. Electronic correlation diagram for $\mathrm{Br}+\mathrm{HI}$ reaction assuming $C_{x_{V}}$ symmetry. The region between the dashed lines is where Hund's case (a) is appropriate (Ref. 55).

ment. In other words, the electronic state structure of the BrHI complex in the geometry resulting from photodetachment of $\mathrm{BrHI}^{-}$is clearly recognizable as that of an I atom slightly perturbed by its interaction with $\mathrm{HBr}$. On the other hand, the vibrational structure in the ground- and excited-state progressions is quite similar. This indicates that, in the geometry probed by photodetachment, the distortion of the $\mathrm{HBr}$ bond is approximately independent of the I atom electronic state, and the groundand spin-orbit excited-state potential energy surfaces are not very different in the $\mathrm{I}+\mathrm{HBr}$ exit valley.

The observation of two, rather than three, electronic states in the photoelectron spectra indicates that the splitting of the $\mathrm{I}\left({ }^{2} \mathrm{P}_{3 / 2}\right)$ state by the $\mathrm{HBr}$ is too small to resolve in our experiment. However, the peaks in the ground-state progression are, on the average, $0.020 \mathrm{eV}$ broader than in the excited state progression $(0.170 \mathrm{eV}$ vs. $0.150 \mathrm{eV})$. This could 

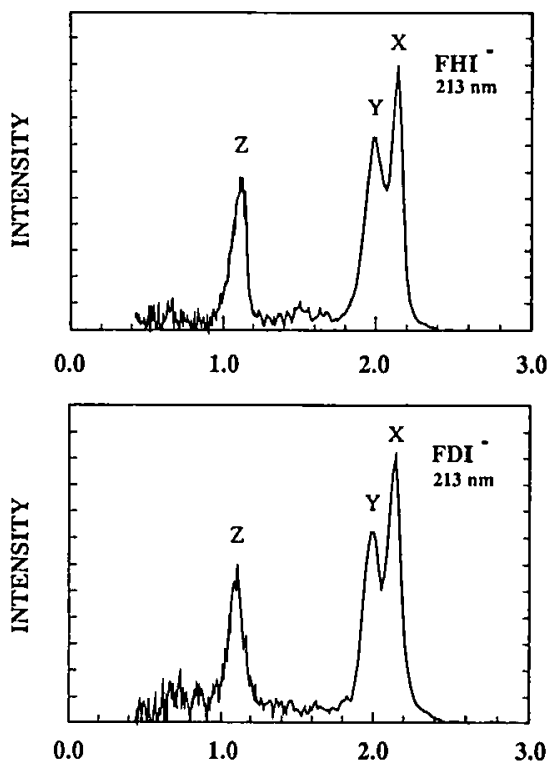

ELECTRON KINETIC ENERGY (eV)

Figure 27. 213-nm photoelectron spectra of $\mathrm{FHI}^{-}$and $\mathrm{FDI}^{-}$(Ref. 55).

mean that each ground-state peak is actually composed, for example, of two $0.150-\mathrm{eV}$ wide peaks split by a $0.020-\mathrm{eV} \mathrm{X}(1 / 2)-\mathrm{I}(3 / 2)$ interval; the sum of two such peaks would yield a single peak in the photoelectron spectrum. A stronger interaction between the halogen atom and HX molecule should yield three distinct electronic states in the photoelectron spectrum. Examples in which this is the case are presented in the next section.

\section{Photoelectron Spectroscopy of $\mathrm{FHI}^{-}$and $\mathrm{FHBr}^{-}$}

The photoelectron spectra of $\mathrm{FHI}^{-}, \mathrm{FHBr}^{-}$, and their deuterated analogs are shown in Figs. 27 and 28 . The proton affinity of $\mathrm{F}^{-}$is substantially higher than that of $\mathrm{Br}^{-}$and $\mathrm{I}^{-}\left(2.07\right.$ and $2.47 \mathrm{eV}$, respectively $\left.{ }^{87}\right)$, and the $\mathrm{F}+\mathrm{HI}$ and $\mathrm{F}+\mathrm{HBr}$ reactions are highly exothermic $(2.815$ and $2.111 \mathrm{eV}$, respectively). Therefore, photodetachment should access the repulsive $\mathrm{HF}+\mathrm{I}(\mathrm{Br})$ product valley in both cases, just as in $\mathrm{BrHI}^{-}$photodetachment. However, although the spectra in Figs. 27 and 28 show resolved features, they are qualitatively different from the $\mathrm{BrHI}^{-} / \mathrm{BrDI}^{-}$spectra in that no isotope shifts are observed. The $\mathrm{FHI}^{-}$and $\mathrm{FDI}^{-}$spectra each show three peaks labelled $X, Y$, and $Z$. These peaks occur at identical energies in both spectra. The $\mathrm{X}-\mathrm{Y}$ spacing is $0.151 \mathrm{eV}\left(1220 \mathrm{~cm}^{-1}\right)$, and the $\mathrm{X}-\mathrm{Z}$ spacing is $1.045 \mathrm{eV}\left(8430 \mathrm{~cm}^{-1}\right)$. Three peaks clearly analogous to these appear in the $\mathrm{FHBr}^{-}$and $\mathrm{FDBr}^{-}$spectra. In both spectra, the $\mathrm{X}$ - 
Figure 28. 213-nm photoelectron spectra of $\mathrm{FHBr}^{-}$and $\mathrm{FDBr}^{-}$.
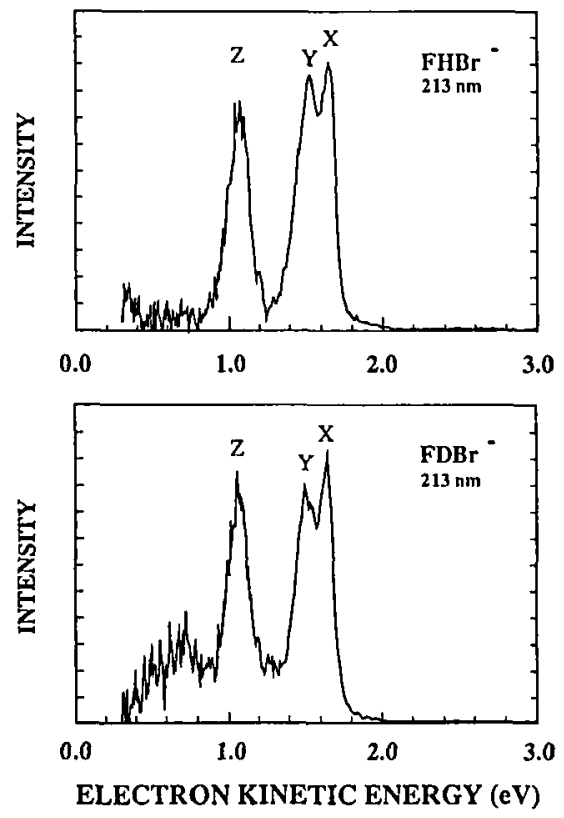

$\mathrm{Y}$ and $\mathrm{X}-\mathrm{Z}$ spacings are $0.121 \mathrm{eV}\left(980 \mathrm{~cm}^{-1}\right)$ and $0.600 \mathrm{eV}\left(4820 \mathrm{~cm}^{-1}\right)$, respectively.

The absence of an isotope shift in these spectra suggests that peaks $X$, $\mathrm{Y}$, and $\mathrm{Z}$ are due to transitions to three distinct electronic states of the neutral complex. In the $\mathrm{FHI}^{-}$spectrum, the $\mathrm{X}-\mathrm{Z}$ spacing is $0.102 \mathrm{eV}$ larger than the spin-orbit splitting in atomic $\mathrm{I}$, while the $\mathrm{X}-\mathrm{Z}$ spacing in the $\mathrm{FHBr}^{-}$spectrum is $0.150 \mathrm{eV}$ larger than the ${ }^{2} \mathrm{P}_{3 / 2}{ }^{2} \mathrm{P}_{1 / 2}$ splitting in atomic $\mathrm{Br}(0.450 \mathrm{eV})$. With reference to the correlation diagram (Fig. 26), the peak pattern in the $\mathrm{FHI}^{-}$and $\mathrm{FHBr}^{-}$spectra is what would be expected if the neutral complex formed by photodetachment can be described by a $\mathrm{Br}$ or I atom interacting with $\mathrm{HF}$. The larger deviation from the atomic spin-orbit splitting in these spectra compared to the $\mathrm{BrHI}^{-}$ spectrum indicates that this electronic interaction is stronger in the $\mathrm{FHBr}$ and FHI complexes. This is consistent with the observation of three distinct electronic states in the $\mathrm{FHI}^{-}$and $\mathrm{FHBr}^{-}$spectra compared to only two in the $\mathrm{BrHI}^{-}$spectrum. The closely spaced $\mathrm{X}$ and $\mathrm{Y}$ peaks are assigned to the two states that result when degeneracy of the $\mathrm{Br}$ or $\mathrm{I}\left({ }^{2} \mathrm{P}_{3 / 2}\right)$ ground state is noticeably split by its interaction with $\mathrm{HF}$.

The $\mathrm{FHI}^{-}$and $\mathrm{FHBr}^{-}$spectra also differ from the $\mathrm{BrHI}^{-}$spectrum in that they do not exhibit a vibrational progression in the $v_{3}$ mode; only $\Delta v_{3}=0$ transitions are observed. For this to occur, the $v_{3}$ potentials for the anion and neutral (see Fig. 21) must be similar near the anion equilibrium 
geometry. A recent $a b$ initio calculation of the $\mathrm{FHBr}^{-}$equilbrium geometry by Sannigrahi and Peyerimhoff ${ }^{89}$ yields $R_{e}(\mathrm{HF})=0.945 \AA$ and $R_{e}(\mathrm{HBr})=1.824 \AA$. The $\mathrm{H}-\mathrm{F}$ separation is very close to $R_{e}=0.917 \AA$ in diatomic $\mathrm{HF}$, while the $\mathrm{H}-\mathrm{Br}$ separation is considerably greater than $R_{e}=$ $1.413 \AA$ in diatomic $\mathrm{HBr} .{ }^{94}$ This means that $\mathrm{FHBr}^{-}$can be thought of as an essentially intact $\mathrm{HF}$ molecule complexed to $\mathrm{Br}^{-}$. Near the equilibrium geometry, $\mathrm{R}_{\mathrm{H}-\mathrm{F}}$ is the appropriate $v_{3}$ coordinate for $\mathrm{FHBr}^{-}$, and the $v_{3}$ potential for $\mathrm{FHBr}^{-}$is very similar to the potential function for HF. The highly asymmetric $a b$ initio structure is reasonable as the proton affinity of $\mathrm{F}^{-}$is much higher than that of $\mathrm{Br}^{-}$. A similar highly asymmetric geometry and $v_{3}$ potential are expected for $\mathrm{FHI}^{-}$.

The lack of a $v_{3}$ progression in the photoelectron spectra therefore means that the neutral $v_{3}$ potentials for the $\mathrm{FHBr}$ and FHI complexes must also resemble the potential for diatomic HF, at least near the mimimum in the potential function. This is a reasonable expectation. The $\mathrm{F}+\mathrm{HI}$ and $\mathrm{F}+\mathrm{HBr}$ reactions are highly exothermic, so the double minimum $v_{3}$ potential should be highly asymmetric with the $X+H F$ product valley side much lower in energy than the $F+H X$ reactant valley side. One therefore expects the lowest few $v_{3}$ levels to look like isolated HF vibrational energy levels. The neutral $v_{3}$ wavefunctions will begin to deviate from diatomic HF wavefunctions only near the energy of the second (reactant) minimum in the $v_{3}$ potential; these highly excited levels have no Franck-Condon overlap with the $v_{3}^{\prime \prime}=0$ levels of $\mathrm{FHBr}^{-}$and $\mathrm{FHI}^{-}$.

In summary, the photoelectron spectra show that in the neutral complex resulting from photodetachment of $\mathrm{FHI}^{-}$and $\mathrm{FHBr}^{-}$, the $\mathrm{HF}$ interacts with the $\mathrm{I}$ or $\mathrm{Br}$ atom strongly enough to noticeably perturb its electronic structure. One is still in the Hund's case (c) regime, since the spin-orbit splitting in the halogen atom is larger than the $X(1 / 2)-I(3 / 2)$ splitting, but the deviation from the energy levels of the isolated halogen atom is noticeably larger than in the BrHI complex. Although the electronic interactions in the $\mathrm{FHI}$ and $\mathrm{FHBr}$ complexes are stronger than in the $\mathrm{BrHI}$ complex, the HF bond is less perturbed in the former systems than the $\mathrm{HBr}$ bond is in the latter, since the $\mathrm{BrHI}^{-}$spectra shows a vibrational progression while the $\mathrm{FHI}^{-}$and $\mathrm{FHBr}^{-}$spectra do not. Finally, we note that although the calculations by Manz and co-workers ${ }^{53,74,96}$ on the $\mathrm{F}+\mathrm{DBr}$ reaction predict strong resonances, the highly excited $v_{3}$ states responsible for the resonances have nearly zero overlap with the anion ground state.

\section{Photoelectron Spectroscopy of $\mathrm{CH}_{3} \mathrm{OHF}^{-}$and $\mathrm{CH}_{3} \mathrm{ODF}^{-}$}

The photoelectron spectra of the asymmetric bihalide anions discussed in Sections V.B and V.C are sensitive to features of the product valley of the potential energy surface for the corresponding neutral reaction, and 
are relatively insensitive to the entrance valley and saddle-point region. This is the case for all of the asymmetric bihalides, not just the ones discussed above. The nature of the product valley in an exothermic reaction is of considerable interest since it plays a major role in the partitioning of excess energy among product degrees of freedom. On the other hand, it was pointed out in Section V.A that photodetachment of $\mathrm{CH}_{3} \mathrm{OHF}^{-}$ should access the reactant valley saddle-point region of the reaction $\mathrm{F}+\mathrm{CH}_{3} \mathrm{OH} \rightarrow \mathrm{HF}+\mathrm{CH}_{3} \mathrm{O}$. This is arguably the most important region of the potential energy surface as it includes the barrier along the minimum energy path and the classical transition state. The $\mathrm{CH}_{3} \mathrm{OHF}^{-} / \mathrm{F}+\mathrm{CH}_{3} \mathrm{OH}$ system is also of interest as it presents an opportunity to extend our method to reactions involving polyatomic species. In this section, the photoelectron spectrum of $\mathrm{CH}_{3} \mathrm{OHF}^{-}$is presented and compared to the asymmetric bihalide spectra.

The $\mathrm{F}+\mathrm{CH}_{3} \mathrm{OH}$ reaction has been the subject of both kinetics ${ }^{95}$ and product state-resolved experiments. ${ }^{96-98}$ Hydrogen abstraction by the $\mathrm{F}$ atom can yield either $\mathrm{CH}_{3} \mathrm{O}$ or $\mathrm{CH}_{2} \mathrm{OH}$. Although $\mathrm{CH}_{2} \mathrm{OH}$ production is more exothermic $(176.6 \mathrm{~kJ} / \mathrm{mol}$ vs. $134.7 \mathrm{~kJ} / \mathrm{mol}$ at $298 \mathrm{~K})$, the $\mathrm{CH}_{3} \mathrm{O}$ channel dominates at room temperature. ${ }^{95}$ In the $\mathrm{CH}_{3} \mathrm{OHF}^{-}$anion, $\mathrm{F}^{-}$ will preferentially bind to the hydroxyl hydrogen since this is considerably more acidic than the methyl hydrogens in $\mathrm{CH}_{3} \mathrm{OH} .{ }^{99}$ Thus, only the transition state region for the $\mathrm{CH}_{3} \mathrm{O}$ channel is probed in our experiment.

The photoelectron spectra of $\mathrm{CH}_{3} \mathrm{OHF}^{-}$and $\mathrm{CH}_{3} \mathrm{ODF}^{-}$at $213 \mathrm{~nm}$ are shown in Fig. 29. The spectra show resolved features, although they are more complex than the spectra of the triatomic anions discussed previously. The product and reactant asymptotes are indicated by the arrows at electron kinetic energies of 2.59 and $1.17 \mathrm{eV}$, respectively. These are based on the $\mathrm{CH}_{3} \mathrm{OH} \cdot \mathrm{F}^{-}$binding energy of $1.28 \mathrm{eV}$ determined by Larson and McMahon. ${ }^{99}$

The most prominent feature in the $\mathrm{CH}_{3} \mathrm{OHF}^{-}$spectrum is the set of four steps with onsets at $2.69,2.24,1.85$, and $1.54 \mathrm{eV}$. The interval between the two highest energy steps is $0.45 \mathrm{eV}\left(3630 \mathrm{~cm}^{-1}\right)$ and decreases to $0.31 \mathrm{eV}\left(2500 \mathrm{~cm}^{-1}\right)$ between the two lowest energy steps. In the $\mathrm{CH}_{3} \mathrm{ODF}^{-}$spectrum, a clear isotope effect is apparent; six more closely spaced steps occur in the same energy range. Thus, the steps appear to correspond to a vibrational progression in the neutral $\mathrm{CH}_{3} \mathrm{OHF}$ complex. The isotope shift and the magnitude of the interval between the steps suggests this progression involves a vibrational mode analogous to the $v_{3}$ mode seen in the bihalide photoelectron spectra in which the light $\mathrm{H}$ atom is vibrating between the much heavier $\mathrm{F}$ atom and $\mathrm{CH}_{3} \mathrm{O}$ group in the neutral complex. The steps occur at energies where only the $\mathrm{CH}_{3} \mathrm{O}+\mathrm{HF}$ products are accessible, but the intervals between the steps are consider- 

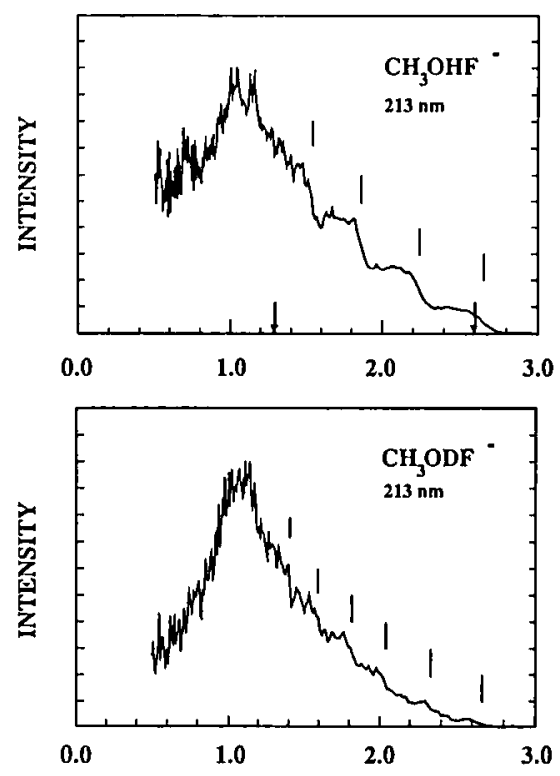

Figure 29. 213-nm photoelectron spectra of $\mathrm{CH}_{3} \mathrm{OHF}^{-}$and $\mathrm{CH}_{3} \mathrm{ODF}^{-}$. Arrows indicate product and reactant asymptotes (see text). Step onsets are indicated with ELECTRON KINETIC ENERGY (eV) short vertical lines.

ably less than the HF fundamental $\left(3958 \mathrm{~cm}^{-1}\right)$. This shows we are close to the transition state region on the $\mathrm{F}+\mathrm{CH}_{3} \mathrm{OH}$ surface.

In contrast to the $\mathrm{BrHI}^{-}$spectrum, the intensity in the $\mathrm{CH}_{3} \mathrm{OHF}^{-}$ spectrum is small at the product asymptote and builds as the reactant asymptote is approached. This is a consequence of the improved FranckCondon overlap between the anion and reactant valley of the neutral potential energy surface. If one approximates $\mathrm{CH}_{3} \mathrm{OHF}{ }^{-} / \mathrm{F}+\mathrm{CH}_{3} \mathrm{OH}$ as a three-atom $\mathrm{AHB}^{-} / \mathrm{A}+\mathrm{HB}$ system, the neutral potential energy surface for the collinear geometry will resemble the surface in Fig. 20, and the effective $v_{3}$ potential will look like the double minimum potential in Fig. 21. However, the minimum in the anion $v_{3}$ potential will lie somewhere under the reactant minimum of the neutral potential. The anion ground state will therefore have poor overlap with the lowest neutral $v_{3}$ levels, as these are localized in the product minimum, but better overlap with the higher $v_{3}$ levels is expected. Thus, the overall intensity distribution in the $\mathrm{CH}_{3} \mathrm{OHF}^{-}$spectrum is reasonable in light of this simple one-dimensional picture.

The step structure in the $\mathrm{CH}_{3} \mathrm{OHF}^{-}$photoelectron spectrum is quite different from the well-resolved peaks in the bihalide spectra. This raises the question of homogeneous vs. inhomogeneous features in these spectra. Since we are photodetaching a polyatomic anion it is possible that other 
modes in addition to the " $v_{3}$ mode" may be active in the photoelectron spectrum, and that the steps represent clumps of unresolved transitions.

Another possibility is that the broad step structure results from the dynamics on the $\mathrm{F}+\mathrm{CH}_{3} \mathrm{OH}$ potential energy surface. The steps occur between the product and reactant asymptotes, and therefore correspond to states of the complex that dissociate to $\mathrm{HF}+\mathrm{CH}_{3} \mathrm{O}$ but not $\mathrm{F}+\mathrm{CH}_{3} \mathrm{OH}$. The dissociation energy of $\mathrm{CH}_{3} \mathrm{OHF}^{-}(1.28 \mathrm{eV})$ is considerably larger than that of $\mathrm{BrHI}^{-}(0.70 \mathrm{eV}),{ }^{57} \mathrm{FHI}^{-}(0.65 \mathrm{eV}),{ }^{100}$ and $\mathrm{FHBr}^{-}$ $(0.74 \mathrm{eV}) .{ }^{100}$ The more tightly bound anion leads one to expect the neutral complex to be more compact. Treating $\mathrm{CH}_{3} \mathrm{OHF}^{-}$as a triatomic linear anion, this means the dashed vertical line in Fig. 20, which in this case corresponds to $R_{\mathrm{O}-\mathrm{F}}$ at the equilibrium geometry of the anion, should be moved in to a smaller value of $x$ than is appropriate for $\mathrm{BrHI}^{-}$photodetachment. At such a value, the product valley is more repulsive, leading one to expect that levels of the complex localized in the product valley will dissociate more rapidly.

On the other hand, since the $\mathrm{F}+\mathrm{CH}_{3} \mathrm{OH}$ reaction is thought to have a small barrier, the reactant valley and saddle-point region of the potential energy surface should be quite flat, and states of the complex localized in this region should have relatively long lifetimes. Indeed, several distinct peaks appear in the $\mathrm{CH}_{3} \mathrm{OHF}^{-}$photoelectron spectrum at electron energies where $\mathrm{F}+\mathrm{CH}_{3} \mathrm{OH}$ reactants are accessible as dissociation products of the complex. The observation of relatively narrow peaks in one region of the spectrum and broad steps in another may therefore be an indication of state-specific dissociation dynamics in the $\mathrm{CH}_{3} \mathrm{OHF}$ complex.

The explanation of the $\mathrm{CH}_{3} \mathrm{OHF}^{-}$spectrum in terms of dissociation dynamics on a collinear three-atom potential energy surface is somewhat of an oversimplification, and it seems likely that the polyatomic nature of the system contributes to the complexity of the spectrum. However, we have recently obtained the photoelectron spectrum of $\mathrm{FHO}^{-}$and found it to be remarkably similar to the $\mathrm{CH}_{3} \mathrm{OHF}^{-}$spectrum. ${ }^{101}$ This suggests that three-atom dynamics may explain a good fraction of the $\mathrm{CH}_{3} \mathrm{OHF}{ }^{-}$ spectrum. This is clearly a subject that warrants further investigation in the near future.

\section{SUMMARY}

This chapter has summarized several studies of the transition state region in bimolecular reactions using negative ion photodetachment. The results presented here show how photoelectron spectroscopy and threshold photodetachment spectroscopy can provide a detailed picture of the spectroscopy and dissociation dynamics of a complex formed in the transi- 
tion state region of a reactive potential energy surface. The higher resolution threshold photodetachment results are of particular interest as they represent the definitive observation of reactive resonances in the $\mathrm{I}+\mathrm{HI}$ reaction. Although much of the work so far has focused on hydrogen exchange between two halogen atoms, the results in Section V.D show that these methods can be applied to polyatomic systems as well. Future applications of these methods will focus on studies of other polyatomic reactions as well as experiments in which reactions are initiated by photodetaching bihalide anions embedded in clusters of known size. ${ }^{102}$

\section{ACKNOWLEDGMENTS}

The photoelectron spectroscopy experiments are supported by the Air Force Office of Scientific Research, Grant. No. AFOSR-91-0084. The threshold photodetachment studies are supported by the Chemistry Division of the Office of Naval Research, Grant. No. N0014-87-K-0495. The authors thank George Schatz, Joel Bowman, and David Tannor for many stimulating discussions. DMN thanks the other members of his research group who contributed to the work presented here: Don Arnold, Caroline Chick, Theofanis Kitsopoulos, Dr. Irene Waller, and Alexandra Weaver.

\section{REFERENCES}

1. P. R. Brooks, Chem. Rev. 88, 407 (1988).

2. A. H. Zewail, Science 242, 1645 (1988); M. Gruebele and A. H. Zewail, Physics Today 43, 24 (1990).

3. N. F. Scherer, L. R. Khundkar, R. B. Bernstein, and A. H. Zewail, J. Chem. Phys. 87, 1451 (1987); N. F. Scherer, C. Sipes, R. B. Bernstein, and A. H. Zewail, J. Chem. Phys. 92, 5239 (1990).

4. M. Dantus, M. J. Rosker, and A. H. Zewail, J. Chem. Phys. 87, 2395 (1987); R. M. Bowman, M. Dantus, and A. H. Zewail, Chem. Phys. Lett. 56, 131 (1989); M. Dantus, R. M. Bowman, M. Gruebele, and A. H. Zewail, J. Chem. Phys. 91,7437 (1989).

5. S. Golub and B. Steiner, J. Chem. Phys. 49, 5191 (1968).

6. C. R. Moylan, J. D. Dodd, C. Han, and J. I. Brauman, J. Chem. Phys. 86, 5350 (1987).

7. M. W. Siegel, R. J. Celotta, J. L. Hall, J. Levine, and R. A. Bennett, Phys. Rev, A 6, 607, 631 (1972).

8. H. Hotop, R. A. Bennett, and W. C. Lineberger, J. Chem. Phys. 58, 2373 (1973).

9. T. N. Kitsopoulos, I. M. Waller, J. G. Loeser, and D. M. Neumark, Chem. Phys. Lett. 159, 300 (1989).

10. K. K. Murray, T. M. Miller, D. G. Leopold, and W. C. Lineberger, J. Chem. Phys. 84, 2520 (1986).

11. S. M. Burnett, A. E. Stevens, C. S. Feigerle, and W. C. Lineberger, Chem. Phys. 
Lett. 100, 124 (1983); K. M. Ervin, J. Ho, and W. C. Lineberger, J. Chem. Phys. 91, 5974 (1989).

12. B. S. Ault, Acc. Chem. Res. 15, 103 (1982) and references therein.

13. D. G. Truhlar and A. Kuppermann, J. Chem. Phys. 52, 384 (1970),

14. S.-F. Wu and R. D. Levine, Mol. Phys. 22, 991 (1971).

15. G. C. Schatz and A. Kuppermann, J. Chem. Phys. 59, 964 (1973); S.-F. Wu, B. R. Johnson, and R. D. Levine, Molec. Phys. 25, 609 (1973).

16. G. C. Schatz, J. M. Bowman, and A. Kuppermann, J. Chem. Phys. 63, 674 (1975).

17. S. L. Latham, J. F. McNutt, R. E. Wyatt, and M. J. Redmon, J. Chem. Phys. 69, 3746 (1978).

18. J. N. L. Connor, W. Jakubetz, and J. Manz, Molec. Phys. 35, 1301 (1978).

19. J. M. Launay and M. Le Dourneuf, J. Phys. B 15, L455 (1982).

20. E. Pollak, J. Chem. Phys. 78, 1228 (1983).

21. D. K. Bondi, J. N. L. Connor, J. Manz, and J. Römelt, Mol. Phys. 50, 467 (1983).

22. A. Kuppermann, in Potential Energy Surface and Dynamics Calculations, D. G. Truhlar, Ed. Plenum, New York, 1981, pp. 375-420.

23. G. C. Schatz and A. Kuppermann, Phys. Rev. Lett. 35, 1266 (1975).

24. M. J. Redmon and R. E. Wyatt, Chem. Phys. Lett. 63, 209 (1979).

25. J. Z. H. Zhang and W. H. Miller, J. Chem. Phys. 91, 1528 (1989); D. E. Manolopoulos and R. E. Wyatt, J. Chem. Phys. 92, 810 (1990); M. Zhao, D. G. Truhlar, D. W. Schwenke, and D. J. Kouri, J. Phys. Chem. 94, 7074 (1990).

26. J.-C. Nieh and J. J. Valentini, Phys. Rev. Lett. 60, 519 (1988).

27. D. M. Neumark, A. M. Wodtke, G. N. Robinson, C. C. Hayden, and Y. T. Lee, J. Chem. Phys. 82, 3045 (1985).

28. R. B. Metz, A. Weaver, S. E. Bradforth, T. N. Kitsopoulos, and D. M. Neumark, J. Phys. Chem, 94, 1377 (1990).

29. L. A. Posey, M. J. DeLuca, and M. A. Johnson, Chem. Phys. Lett. 131, 170 (1986).

30. M. A. Johnson and W. C. Lineberger, in Techniques in Chemistry, Vol. 20, J. M. Farrar and W. H. Saunders, Jr., Eds., Wiley, New York, 1988, pp. 591-635.

31. W. C. Wiley and I. H. Maclaren, Rev. Sci. Instrum. 26, 1150 (1955).

32. K. Müller-Dethlefs, M. Sander, and E. W. Schlag, Z. Naturforsch. 39a, 1089 (1984); Chem. Phys. Lett. 12, 291 (1984).

33. J. M. B. Bakker, J. Phys. E 6, 785 (1973); 7, 364 (1974).

34. G. C. Schatz, J. Phys. Chem. 94, 6157 (1990).

35. G. C. Schatz, J. Chem. Phys. 90, 1237 (1989).

36. G. C. Schatz, J. Chem. Phys. 90, 4847 (1989).

37. G. C. Schatz, Chem. Phys. Lett. 150, 92 (1988).

38. K. Kawaguchi, J. Chem. Phys. 88, 4186 (1988).

39. J. Z. H. Zhang and W. H. Miller, J. Chem. Phys. 92, 1811 (1990).

40. R. Steckler, D. G. Truhlar, and B. C. Garrett, J. Chem. Phys. 82, 5499 (1985).

41. R. A. Kendall, J. A. Nichols, and J. Simons, J. Phys. Chem. 94, 1074 (1991).

42. A. Weaver, R. B. Metz, S. E. Bradforth, and D. M. Neumark, J. Chem. Phys. 93, 5352 (1990); A. Weaver and D. M. Neumark, Faraday Discuss. Chem. Soc. 91, (1991).

43. B. Gazdy and J. M. Bowman, J. Chem. Phys. 91, 4615 (1989). 
44. J. M. Bowman, Adv. Chem. Phys. 61, 115 (1985).

45. A. B. Sannigrahi and S. D. Peyerimhoff, J. Mol. Struct. 165, 55 (1988).

46. S. Ikuta, T. Saitoh, and O. Nomura, J. Chem. Phys. 93, 2530 (1990).

47. J. M. Bowman, B. Gazdy, and Q. Sun, J. Chem. Soc. Faraday Trans. 86, 1737 (1990).

48. J. M. Bowman and B. Gazdy, J. Phys. Chem. 93, 5129 (1989).

49. J. Römelt, Chem. Phys. 79, 197 (1983).

50. E. J. Heller, J. Chem. Phys. 68, 3891 (1978).

51. R. Kosloff, J. Phys. Chem. 92, 2087 (1988).

52. R. H. Bisseling, R. Kosloff, and J. Manz, J. Chem. Phys. 83, 993 (1985).

53. R. H. Bisseling, P. L. Gertitschke, R. Kosloff, and J. Manz, J. Chem. Phys, 88, 6191 (1988).

54. A. J. Lorquet, J. C. Lorquet, J. Delwiche, and M. J. Hubin-Franskin, J. Chem. Phys. 76, 4692 (1982).

55. S. E. Bradforth, A. Weaver, D. W. Arnold, R. B. Metz, and D. M. Neumark, J. Chem. Phys. 92, 7205 (1990).

56. D. Kosloff and R. Kosloff, J. Comput. Phys. 52, 35 (1983).

57. G. Caldwell and P. Kebarle, Can. J. Chem. 63, 1399 (1985).

58. H. Hotop and W. C. Lineberger, J. Phys. Chem. Ref. Data 14, 731 (1985).

59. P. Botschwina and W. Meyer, Chem. Phys. Lett. 44, 449 (1976).

60. B. C. Garrett, D. G. Truhlar, A. F. Wagner, and T. H. Dunning, Jr., J. Chem. Phys. 78, 4400 (1983).

61. K. Kawaguchi and E. Hirota, J. Chem. Phys. 87, 6838 (1987).

62. V. Bondybey, G. C. Pimentel, and P. N. Noble, J. Chem. Phys. 55, 540 (1971); D.

E. Milligan and M. E. Jacox, J. Chem. Phys. 55, 2550 (1971).

63. See Ref. 28 for the explicit form of this one-dimensional potential.

64. N. C. Firth and R. Grice, J. Chem. Soc. Faraday Trans. 2. 87, 1023 (1987).

65. R. B. Metz, T. Kitsopoulos, A. Weaver, and D. M. Neumark, J. Chem. Phys. 88, 1463 (1988).

66. K. Yamashita and K. Morokuma, J. Chem. Phys. 93, 3716 (1990).

67. R. J. Saykally, Science 239, 157 (1988).

68. J. Manz and J. Römelt, Chem. Phys. Lett. 81, 179 (1981).

69. A. Weaver, R. B. Metz, S. E. Bradforth, and D. M. Neumark, J. Phys. Chem. 92, 5558 (1988).

70. J. Manz, R. Meyer, E. Pollak, and J. Römelt, Chem. Phys. Lett. 93, 184 (1982); E. Pollak, Chem. Phys. Lett. 94, 85 (1983); J. Manz, R. Meyer, E. Pollak, J. Römelt, and H. H. R. Schor, Chem. Phys. 83, 333 (1984).

71. D. C. Clary and J. N. L. Connor, Chem. Phys. Lett. 94, 81, 1983; J. Phys. Chem. 88, 2758 (1984).

72. I. M. Waller, T. N. Kitsopoulos, and D. M. Neumark, J. Phys. Chem. 94, 2240 (1990).

73. G. C. Schatz, private communication.

74. P. L. Gertitschke, J. Manz, J. Römelt, H. H. R. Schor, J. Chem. Phys. 83, 208 (1985).

75. D. Tannor, private communication.

76. C. Kubach, Chem. Phys. Letr. 164, 475 (1989); C. Kubach, G. N. Vien, and M. Richard-Viard, J. Chem. Phys. 94, 1929 (1991). 
77. K. G. Anlauf, P. J. Kuntz, D. H. Maylotte, P. D. Pacey, and J. C. Polanyi, Disc. Faraday Soc. 44, 183 (1967); D. H. Maylotte, J. C. Polanyi, and K. B. Woodall, $J$. Chem. Phys. 57, 1547 (1972).

78. N. Jonathan, C. M. Melliar-Smith, S. Okuda, D. H. Slater, and D. Timlin, Molec. Phys. 22, 4 (1971).

79. P. J. Kuntz, E. M. Nemeth, J. C. Polanyi, S. D. Rosner, and C. E. Young, J. Chem. Phys. 44, 1168 (1966); C. A. Parr, J. C. Polanyi, and W. H. Wong, J. Chem. Phys. 58, 5 (1973).

80. I. W. M. Smith, Chem. Phys. 20, 437 (1977).

81. J. C. Brown, H. E. Bass, and D. L. Thompson, J. Phys. Chem. 81, 479 (1977).

82. P. Beadle, M. R. Dunn, N. B. H. Jonathan, J. P. Liddy, and J. C. Naylor, J. Chem. Soc. Faraday Trans. 274, 2170 (1978).

83. M. Broida and A. Persky, Chem. Phys. 133, 405 (1989).

84. M. Baer, J. Chem. Phys. 62, 305 (1975).

85. J. A. Kaye and A. Kuppermann, Chem. Phys. Lett. 92, 574 (1982).

86. R. A. Fischer, P. L. Gertitschke, J. Manz, and H. H. R. Schor, Chem. Phys. Lett. 156, 100 (1989); J. Manz and J. Römelt, J. Chem. Soc. Faraday Trans. 86, 1689 (1990).

87. S. G. Lias, J. E. Bartmess, J. F. Leibman, J. L. Holmes, R. D. Levin, and W. G. Mallard, J. Phys. Chem. Ref. Data 17, Suppl. No. 1 (1988).

88. C. M. Ellison and B. S. Ault, J. Phys. Chem. 83, 832 (1979).

89. A. B. Sannigrahi and S. D. Peyerimhoff, Chem. Phys. Lett. 112, 267 (1984); 164, 348 (1989).

90. C. C. Mei and C. B. Moore, J. Chem. Phys. 70, 1759 (1979); D. A. Dolson and S. R. Leone, J. Chem. Phys. 77, 4009 (1982).

91. These curves are the eigenvalues of Eq. (11); the DIVAH correction (Eq. 18) is not included. Manz and co-workers (Ref. 86) have shown the $Q_{v_{3} v_{3}}$ term in Eq. (18) becomes large near avoided crossings of the adiabatic curves $\epsilon_{v_{3}}(\rho)$.

92. K. Bergmann, S. R. Leone, and C. B. Moore, J. Chem. Phys. 63, 4161 (1975).

93. J. W. Hepburn, K. Liu, R. G. Macdonald, F. J. Northrup, and J. C. Polanyi, J. Chem. Phys. 75, 3353 (1981).

94. K. P. Huber and G. Herzberg, Molecular Spectra and Molecular Structure IV. Constants of Diatomic Molecules, Van Nostrand, New York, 1979.

95. J. A. McCaulley, N. Kelly, M. F. Golde, and F. Kaufman, J. Phys. Chem. 93, 1014 (1989).

96. R. G. MacDonald, J. J. Sloan, and P. T. Wassell, Chem. Phys. 41, 201 (1979).

97. B. Dill and H. Heydtmann, Chem. Phys. 54, 9 (1980); M. A. Wickramaaratchi, D. W. Setser, H. Hildebrandt, B. Köbitzer, and H. Heydtmann, Chem. Phys. 94, 109 (1985).

98. B. S. Agrawalla and D. W. Setser, J. Phys. Chem. 88, 657 (1984); J. Chem. Phys. 90, 2450 (1986).

99. J. W. Larson and T. B. McMahon, J. Am. Chem. Soc. 105, 2944 (1983).

100. J. L. Larson and T. B. McMahon, Inorg. Chem. 23, 2029 (1984).

101. S. E. Bradforth, R. B. Metz, A. Weaver, and D. M. Neumark, J. Phys. Chem. 95 (in press). 
102. D. M. Neumark, in The Physics of Electronic and Atomic Collisions: XVI International Conference (AIP Conference Proceedings 205), A. Dalgarno, R. S. Freund, P. M. Koch, M. S. Lubell, and T. B. Lucatorto, Eds., American Institute of Physics, New York, 1990, pp. 33-48. 